\title{
Turbulent collision-coalescence in maritime shallow convection
}

\author{
A. A. Wyszogrodzki ${ }^{1}$, W. W. Grabowski ${ }^{1}$, L.-P. Wang ${ }^{2}$, and O. Ayala ${ }^{2,3}$ \\ ${ }^{1}$ National Center for Atmospheric Research, Boulder, Colorado, USA \\ ${ }^{2}$ Department of Mechanical Engineering, University of Delaware, Newark, Delaware, USA \\ ${ }^{3}$ Department of Engineering Technology, Old Dominion University, Norfolk, Virginia, USA
}

Correspondence to: W. W. Grabowski (grabow@ ncar.ucar.edu)

Received: 4 February 2013 - Published in Atmos. Chem. Phys. Discuss.: 8 April 2013

Revised: 2 July 2013 - Accepted: 17 July 2013 - Published: 27 August 2013

\begin{abstract}
This paper discusses cloud simulations aiming at quantitative assessment of the effects of cloud turbulence on rain development in shallow ice-free convective clouds. Cloud fields from large-eddy simulations (LES) applying bin microphysics with the collection kernel enhanced by cloud turbulence are compared to those with the standard gravitational collection kernel. Simulations for a range of cloud condensation nuclei $(\mathrm{CCN})$ concentrations are contrasted. Details on how the parameterized turbulent collection kernel is used in LES simulations are presented. Because of the disparity in spatial scales between the bottom-up numerical studies guiding the turbulent kernel development and the top-down LES simulations of cloud dynamics, we address the consequence of the turbulence intermittency in the unresolved range of scales on the mean collection kernel applied in LES. We show that intermittency effects are unlikely to play an important role in the current simulations. Highly-idealized single-cloud simulations are used to illustrate two mechanisms that operate in cloud field simulations. First, the microphysical enhancement leads to earlier formation of drizzle through faster autoconversion of cloud water into drizzle, as suggested by previous studies. Second, more efficient removal of condensed water from cloudy volumes when a turbulent collection kernel is used leads to an increased cloud buoyancy and enables clouds to reach higher levels. This is the dynamical enhancement. Both mechanisms operate in the cloud field simulations. The microphysical enhancement leads to the increased drizzle and rain inside clouds in simulations with high CCN. In low-CCN simulations with significant surface rainfall, dynamical enhancement leads to a larger contribution of deeper clouds to the entire cloud population, and results in a dramatically increased mean surface
\end{abstract}

rain accumulation. These results call for future modeling and observational studies to corroborate the findings.

\section{Introduction}

Cloud droplets grow by the diffusion of water vapor up to sizes where collision-coalescence among the droplets begins to move the condensed water into drizzle drops and eventually into rain drops. Observed rapid onset of rain in shallow clouds in the tropics and subtropics is often difficult to explain with the classical droplet growth theory, and several mechanisms that may accelerate the onset have been considered in the past; see Cooper et al. (2013) and references therein. The mechanisms include the impact of giant and ultra-giant cloud condensation nuclei $(\mathrm{CCN})$ that can grow by the water vapor diffusion into drizzle sizes, cloud entrainment (that can lead to a significant broadening of the cloud droplet spectrum in diluted volumes and thus promote droplet collisions), and effects of cloud turbulence. The latter includes the effects on relative motion of droplets, concentration fluctuations, and collision efficiencies (Khain et al., 2000; Franklin et al., 2005; Wang et al., 2005). The rate of droplet collisions is controlled by the gravitational mechanism for drizzle/rain drops of radii larger than $50 \mu \mathrm{m}$, but air turbulence can significantly enhance the collision rate for cloud droplets with radii between 10 and $30 \mu \mathrm{m}$ and for intermediate droplets/drops with radii between 30 to $50 \mu \mathrm{m}$ (Grabowski and Wang, 2013).

Effects of air turbulence on geometric collision rates and on collision efficiency have been studied applying the direct numerical simulation (DNS), and accounting for the dropletsize dependence (which in turn determines the response time 
and settling velocity), the strength of air turbulence (i.e., the dissipation rate, Reynolds number, etc.), the gravity force acting on the droplets, and interactions of droplets with the air turbulence (e.g., Franklin et al., 2005; Wang et al., 2005, 2006b; Ayala et al., 2008a, b). The effects of turbulence on the collection kernel include increased relative motion due to differential acceleration and shear effects (Wang et al., 2000; Zhou et al., 2001), the increased average pair density due to local concentration enhancement (Wang et al., 2000; Zaichik and Alipchenkov, 2003; Zaichik et al., 2003), selective alterations of the settling rate by turbulence (Wang and Maxey, 1993; Dávila and Hunt, 2001), and enhanced collision efficiency (Pinsky et al., 1999; Wang et al., 2005, 2008; Ayala et al., 2007). Many questions remain to be answered regarding the true quantitative level of the above effects (Grabowski and Wang, 2013).

Wang et al. (2005) developed a general kinematic formulation that describes the droplet collection kernel in turbulent air with hydrodynamic interactions and gravitational settling. One way to formulate the turbulent collection kernel is to combine a turbulent geometric kernel (Ayala et al., 2008b), gravitational collision efficiency (Hall, 1980), and a collision-efficiency enhancement factor by air turbulence (Wang et al., 2008). A hybrid direct numerical simulation (HDNS, Ayala et al., 2007) has been used to study the turbulent geometric kernel and the collision-efficiency enhancement factor in Wang et al. $(2005,2008)$ and (Ayala et al. (2008a). Ayala et al. (2008b) developed a comprehensive theory for turbulent geometric collection kernel, which was used in Xue et al. (2008) to study the impact of air turbulence on warm rain initiation. It was found that the air turbulence can accelerate the growth of cloud droplets by speeding up the autoconversion phase, thus shorten the time for the formation of drizzle drops by up to $40 \%$ relative to the Hall gravitational collection kernel (Xue et al., 2008; Grabowski and Wang, 2009).

The complexity of droplet interactions with the turbulent flow and limitations of cloud measurements result in uncertain estimates of the level of collision-rate enhancement by air turbulence (Grabowski and Wang, 2013). Additional effects can also widen the droplet spectrum and promote collisional growth. For instance, $\mathrm{CCN}$ differences in marine and continental environments are primarily responsible for microphysical differences between clean and polluted clouds (Pruppacher and Klett, 1997). Moreover, for a given cloud, updraft strength varies significantly across the cloud base and affects the number of activated droplets. Finally, in shallow cumuli, $\mathrm{CCN}$ are activated above the cloud base and lead to the observed approximately constant-with-height mean concentration of cloud droplets despite a significant dilution of such clouds by entrainment (e.g., Slawinska et al., 2012; Wyszogrodzki et al., 2011).

In this paper, we focus on the effects of turbulent acceleration of rain formation in a field of simulated shallow cumulus clouds. Since rain development depends on the cloud depth as well as on the CCN characteristics, we perform a set of model simulations applying a model setup that ensures approximately constant cloud field depth and varying CCN concentrations. Cloud field simulations applying either gravitational or turbulent collection kernels are compared. We apply a large-eddy simulation (LES) fluid flow model (used previously in bulk microphysics simulations discussed in Grabowski, 2007; Jarecka et al., 2009; Slawinska et al., 2012) coupled to the bin microphysics scheme used in studies reported in Grabowski and Wang (2009), Grabowski et al. (2011) and Wyszogrodzki et al. (2011). Our study is similar to the one reported in Seifert et al. (2010) except that we apply a bin microphysics scheme rather than a doublemoment bulk scheme used in Seifert et al. (2010). Formulation of the turbulent enhancement of the traditional gravitational collection kernel is discussed in the next section. Because of computational limitations, a LES model gridbox has to be significantly larger than the volume of the computational domain applied in DNS and HDNS studies guiding the development of the turbulent collection kernel. It follows that the intermittency of the dissipation rate in the physical space needs to be considered before the enhancement is applied in the LES study. This aspect is discussed in Sect. 3. Section 4 provides a brief description of the LES model and details of the model setup. Model results are discussed in Sect. 5. A brief summary in Sect. 6 concludes the paper.

\section{Formulation of gravitational and turbulent collection kernels}

The impact of turbulent collisions on warm-rain processes is evaluated by comparing simulations that apply the turbulent collection kernel with simulations using the classical gravitational kernel. The gravitational collection kernel is given by

$K_{i j}=\pi\left(a_{i}+a_{j}\right)^{2}\left|v_{i}^{\mathrm{t}}-v_{j}^{\mathrm{t}}\right| E_{i j}^{\mathrm{g}}$,

where $E_{i j}^{\mathrm{g}}$ is the gravitational collision efficiency of droplets with radii $a_{i}$ and $a_{j}$ in a quiescent background air, and $v_{i}^{\mathrm{t}}$ and $v_{j}^{\mathrm{t}}$ are droplet sedimentation (terminal) velocities. Collision efficiencies are obtained by linearly interpolating tabulated values given in Hall (1980). Terminal velocities are prescribed according to Beard (1976), as given in Pruppacher and Klett (1997).

The turbulent collection kernel combines the analytical parameterization of the turbulent geometric collection kernel of Ayala et al. (2008b) with the collision-efficiency enhancement factor obtained from HDNS (Wang et al., 2005, 2008; Ayala et al., 2007). Namely, the turbulent collection kernel is expressed as

$K_{i j}=K_{i j}^{\mathrm{tg}} E_{i j}^{\mathrm{g}} \eta_{\mathrm{E}}$

where the turbulent geometric collection kernel $K_{i j}^{\mathrm{tg}}$ is obtained when droplet-droplet local aerodynamic interaction is 
Table 1. The enhancement factor $\eta_{\mathrm{E}}$ of the collision efficiency. The upper/lower part of the table is for $\epsilon=100 / 400 \mathrm{~cm}^{2} \mathrm{~s}^{-3}$.

\begin{tabular}{rccccccc}
\hline$a_{2} / a_{1}$ & $a_{1}=10 \mu \mathrm{m}$ & $20 \mu \mathrm{m}$ & $30 \mu \mathrm{m}$ & $40 \mu \mathrm{m}$ & $50 \mu \mathrm{m}$ & $60 \mu \mathrm{m}$ & $100 \mu \mathrm{m}$ \\
\hline 0.0 & 1.74 & 1.74 & 1.773 & 1.49 & 1.207 & 1.207 & 1.0 \\
0.1 & 1.46 & 1.46 & 1.421 & 1.245 & 1.069 & 1.069 & 1.0 \\
0.2 & 1.32 & 1.32 & 1.245 & 1.123 & 1.000 & 1.000 & 1.0 \\
0.3 & 1.250 & 1.250 & 1.148 & 1.087 & 1.025 & 1.025 & 1.0 \\
0.4 & 1.186 & 1.186 & 1.066 & 1.060 & 1.056 & 1.056 & 1.0 \\
0.5 & 1.045 & 1.045 & 1.000 & 1.014 & 1.028 & 1.028 & 1.0 \\
0.6 & 1.070 & 1.070 & 1.030 & 1.038 & 1.046 & 1.046 & 1.0 \\
0.7 & 1.000 & 1.000 & 1.054 & 1.042 & 1.029 & 1.029 & 1.0 \\
0.8 & 1.223 & 1.223 & 1.117 & 1.069 & 1.021 & 1.021 & 1.0 \\
0.9 & 1.570 & 1.570 & 1.244 & 1.166 & 1.088 & 1.088 & 1.0 \\
1.0 & 20.3 & 20.3 & 14.6 & 8.61 & 2.60 & 2.60 & 1.0 \\
\hline 0.0 & 4.976 & 4.976 & 3.593 & 2.519 & 1.445 & 1.445 & 1.0 \\
0.1 & 2.984 & 2.984 & 2.181 & 1.691 & 1.201 & 1.201 & 1.0 \\
0.2 & 1.988 & 1.988 & 1.475 & 1.313 & 1.150 & 1.150 & 1.0 \\
0.3 & 1.490 & 1.490 & 1.187 & 1.156 & 1.126 & 1.126 & 1.0 \\
0.4 & 1.249 & 1.249 & 1.088 & 1.090 & 1.092 & 1.092 & 1.0 \\
0.5 & 1.139 & 1.139 & 1.130 & 1.091 & 1.051 & 1.051 & 1.0 \\
0.6 & 1.220 & 1.220 & 1.190 & 1.138 & 1.086 & 1.086 & 1.0 \\
0.7 & 1.325 & 1.325 & 1.267 & 1.165 & 1.063 & 1.063 & 1.0 \\
0.8 & 1.716 & 1.716 & 1.345 & 1.223 & 1.100 & 1.100 & 1.0 \\
0.9 & 3.788 & 3.788 & 1.501 & 1.311 & 1.120 & 1.120 & 1.0 \\
1.0 & 36.52 & 36.52 & 19.16 & 22.80 & 26.0 & 26.0 & 1.0 \\
\hline & & & & & & &
\end{tabular}

not considered. In this case, the disturbance flows induced by other droplets are excluded when the motion of a given droplet is solved. The collision efficiency of droplets in a quiescent background air $E_{i j}^{\mathrm{g}}$ is as in Eq. (1). The ratio of turbulent collision efficiency to $E_{i j}^{\mathrm{g}}$ is represented by the relative enhancement factor $\eta_{\mathrm{E}}$ (Wang et al., 2005), which depends on the flow dissipation rate $\epsilon$. The collision-efficiency enhancement factor $\eta_{\mathrm{E}}$ is interpolated from the HDNS data for two intensities of turbulence ( $\epsilon$ of 100 and $400 \mathrm{~cm}^{2} \mathrm{~s}^{-3}$ ) shown in Table 1. Assuming $\eta_{\mathrm{E}}=1$ for $\epsilon=0$, the enhancement factors for other dissipation rates are derived by either interpolation or extrapolation. Specifically, we follow an approach used in Seifert et al. (2010) and calculate $\eta_{\mathrm{E}}$ as follows:

- for $0 \leq \epsilon \leq 100 \mathrm{~cm}^{2} \mathrm{~s}^{-3}$, a linear interpolation between $\eta_{\mathrm{E}}=1$ for $\epsilon=0$ and the value of $\eta_{\mathrm{E}}$ for $\epsilon=$ $100 \mathrm{~cm}^{2} \mathrm{~s}^{-3}$ is used;

- for $100<\epsilon \leq 400 \mathrm{~cm}^{2} \mathrm{~s}^{-3}$, a linear interpolation between the values of $\eta_{\mathrm{E}}$ at $\epsilon=100 \mathrm{~cm}^{2} \mathrm{~s}^{-3}$ and $\epsilon=$ $400 \mathrm{~cm}^{2} \mathrm{~s}^{-3}$ is used;

- for $400<\epsilon \leq 600 \mathrm{~cm}^{2} \mathrm{~s}^{-3}$, a linear extrapolation from the values of $\eta_{\mathrm{E}}$ at $\epsilon=100 \mathrm{~cm}^{2} \mathrm{~s}^{-3}$ and $400 \mathrm{~cm}^{2} \mathrm{~s}^{-3}$ is used;

- for $\epsilon>600 \mathrm{~cm}^{2} \mathrm{~s}^{-3}$, the extrapolated value at $\epsilon=$ $600 \mathrm{~cm}^{2} \mathrm{~s}^{-3}$ is used.
The turbulent geometric collection kernel $K_{i j}^{\mathrm{tg}}$ is given by the kinematic formulation (Wang et al., 2005):

$K_{i j}^{\mathrm{tg}}=2 \pi R^{2}\left\langle\left|\boldsymbol{w}_{r}(r=R)\right|\right\rangle g_{i j}(r=R)$,

where $R=a_{i}+a_{j}$ is the geometric collision radius, the radial relative velocity $\boldsymbol{w}_{r}$ is defined in terms of the center-to-center separation vector $\boldsymbol{r}$ (pointing from a droplet of radius $a_{j}$ to a droplet of radius $a_{i}$ ), the velocity $V_{i}$ of the $a_{i}$ droplet, and the velocity $V_{j}$ of the $a_{j}$ droplet as

$\boldsymbol{w}_{r}=\boldsymbol{r} \cdot\left(V_{i}-V_{j}\right) / r$

with $r=|\boldsymbol{r}|$. The additional factor $g_{i j}$ is the radial distribution function that measures the effect of preferential concentration on the pair number density at separation $r=$ $R$. Both $\left\langle\left|\boldsymbol{w}_{r}\right|\right\rangle$ and $g_{i j}$ in Eq. (3) are computed without local aerodynamic interaction. The kinematic formulation of Eq. (3) has been validated against dynamic collision rates from DNS/HDNS for both ghost droplets and aerodynamically-interacting droplets in a turbulent air flow; see Wang et al. (2005) and Ayala et al. (2008a). Ayala et al. (2008b) developed parameterizations for both $\left\langle\left|\boldsymbol{w}_{r}\right|\right\rangle$ and $g_{i j}$, guided by DNS data. It should be noted that the parameterizations consider the effects of flow Reynolds number that cannot be fully represented by HDNS. For example, the parameterization for $\left\langle\left|\boldsymbol{w}_{r}\right|\right\rangle$ makes use of velocity correlations that are valid for both the dissipation subrange and the energy-containing subrange of turbulence. The intermittency of small-scale turbulent fluctuations was incorporated 
into the model for $g_{i j}$ following Chun et al. (2005). The detailed expression for $K_{i j}^{\mathrm{tg}}$ and $g_{i j}$ can be found in Ayala et al. (2008b).

\section{Effects of the dissipation intermittency on the turbulent collection kernel}

Due to computational limitations, the DNS domain size is currently limited to about $1 \mathrm{~m}$ or less, and only the subdissipation and dissipation scales as well as a limited range of inertial-range scales can be explicitly resolved. The underlying assumption in DNS is that the pair-statistics relevant to turbulent collision-coalescence are governed mostly by the dissipation range scales because the droplet Stokes number (i.e., the ratio between the droplet inertial response time and the flow Kolmogorov time) is typically on the order of one or less. Such a DNS domain size is significantly smaller than the typical grid length $(10-100 \mathrm{~m})$ in atmospheric LES targeting clouds. The limited domain size implies a limited HDNS Taylor microscale Reynolds number, $\sim 500$ or less. This is one to two orders of magnitude lower than what is found in cumulus clouds.

Because of these limitations, there are two general issues that must be resolved. First, one must ensure that the DNSguided collection kernel is independent of the domain size and the large-scale forcing algorithm. Indeed, for droplets smaller than $\sim 30 \mu \mathrm{m}$ in radius, such a convergence has been demonstrated (Rosa et al., 2011, 2013). The parameterization of the turbulent geometric collection kernel of Ayala et al. (2008b) applies a description of the two-point twotime fluid velocity correlation that is valid for much higher flow Reynolds numbers than in DNS. This partially removes the limitation of the small flow Reynolds number in DNS. However, larger droplets have larger settling velocity, and the crossing-trajectory effect combined with droplet inertia could imply a larger range of flow scales affecting the pair relative statistics. DNS data at higher resolutions and larger domain sizes are still needed to validate the theoretical parameterization.

The second issue concerns increasing intermittency of the local dissipation rate with increasing flow Reynolds number. It is well known that the local flow dissipation rate takes more extreme values in small regions when the flow Reynolds number is increased. This is reflected in the higher velocity derivative flatness and more extended tail in PDF of the local dissipation rate and local fluid acceleration (e.g., Sreenivasan and Antonia, 1997; Pinsky and Khain, 2004). Since the local collection kernel depends on the local flow dissipation, a question arises as to whether the different levels of flow intermittency in DNS computational domain and LES grid volume affect the average collection kernel. In practical terms, the LES gridbox consists of thousands to millions of DNS domains, with a distribution of eddy dissipation rates in each DNS domain that average out to the mean dissipation rate of the LES gridbox. Since the turbulent collection kernel derived from DNS is nonlinear in the dissipation rate, simply using the collection kernel based on the LES-derived average dissipation rate may not yield the same answer as averaging DNS kernels with the distribution of the dissipation rates.

More specifically, the latter issue can be formulated as follows. Let $l_{1}$ be the DNS domain size and $l_{2}$ be the gridlength in LES, with $l_{2}>l_{1}$ and both $l_{1}$ and $l_{2}$ falling into the inertial subrange. Moreover, let $\epsilon_{1}$ and $\epsilon_{2}$ denote the flow dissipation rate averaged over a volume of size $l_{1}$ and $l_{2}$, respectively. If the collection kernel derived from DNS is $K_{i j}\left(\epsilon_{1}, u^{\prime}\right)$, then the average collection kernel in the LES grid volume, taking into account of the dissipation intermittency, can be expressed as

$\left\langle K_{i j}\right\rangle \equiv \int K_{i j}\left(\epsilon_{1}, u^{\prime}\left(l_{2}, \epsilon_{1}\right)\right) f\left(\ln \frac{\epsilon_{1}}{\epsilon_{2}} ; \frac{l_{2}}{l_{1}}\right) d \ln \frac{\epsilon_{1}}{\epsilon_{2}}$,

where $f\left(\ln \epsilon_{1} / \epsilon_{2} ; l_{2} / l_{1}\right)$ is the PDF of $\ln \epsilon_{1} / \epsilon_{2}$ for a given $l_{2} / l_{1}$, and in the integrand $u^{\prime}$ is estimated by $u^{\prime} \approx\left(2 \epsilon_{1} l_{2}\right)^{1 / 3}$. There are two reasons why $l_{2}$ is used in estimating $u^{\prime}$. First, the collection kernel is contributed by both resolved and subgrid velocity fluctuations in the LES grid box. Second, $K_{i j}$ inside the integrand is a derived model already extending the level of velocity fluctuations in the DNS domain to the level of velocity fluctuations in clouds, and the only aspect that was not modeled is the added intermittency of the local dissipation rate at cloud Reynolds numbers.

The Kolmogorov refined similarity theory (Kolmogorov, 1962) implies that the PDF of $f$ is Gaussian with a variance of $\sigma_{i j}^{2}=\mu \ln \left(l_{2} / l_{1}\right)$, where $\mu$ is a universal parameter. The mean of the distribution can be determined then by the fact that the mean of $\epsilon_{1}$ according to the prescribed PDF is $\epsilon_{2}$. Therefore, the PDF takes the following form:

$$
f\left(\ln \frac{\epsilon_{1}}{\epsilon_{2}} ; \frac{l_{2}}{l_{1}}\right)=\frac{1}{\sqrt{2 \pi} \sigma_{12}} \exp \left\{-\frac{\left[\ln \left(\epsilon_{1} / \epsilon_{2}\right)+\sigma_{12}^{2} / 2\right]^{2}}{2 \sigma_{12}^{2}}\right\},
$$

where the following consistency condition is enforced

$$
\int_{0}^{\infty} \epsilon_{1} f\left(\ln \frac{\epsilon_{1}}{\epsilon_{2}}\right) d\left(\ln \frac{\epsilon_{1}}{\epsilon_{2}}\right)=\epsilon_{2}
$$

A review of experimental data (Sreenivasan and Kailasnath, 1993) shows that the intermittency exponent $\mu$ takes a value of $\mu=0.25 \pm 0.05$, so the PDF is now completely specified in terms of $\epsilon_{2} / \epsilon_{1}$ and $l_{2} / l_{1}$ ratios. This formulation provides a method to evaluate the effect of dissipation intermittency on $K_{i j}\left(\epsilon_{2}, u^{\prime}\right)$. Specifically, we ask whether the ratio

$\mathcal{R}\left(\frac{l_{2}}{l_{1}}, \epsilon_{2}\right)=\frac{\left\langle K_{i j}\right\rangle}{K_{i j}\left(\epsilon_{2}, u^{\prime}\left(l_{2}, \epsilon_{2}\right)\right)}$

is close to one or not. 


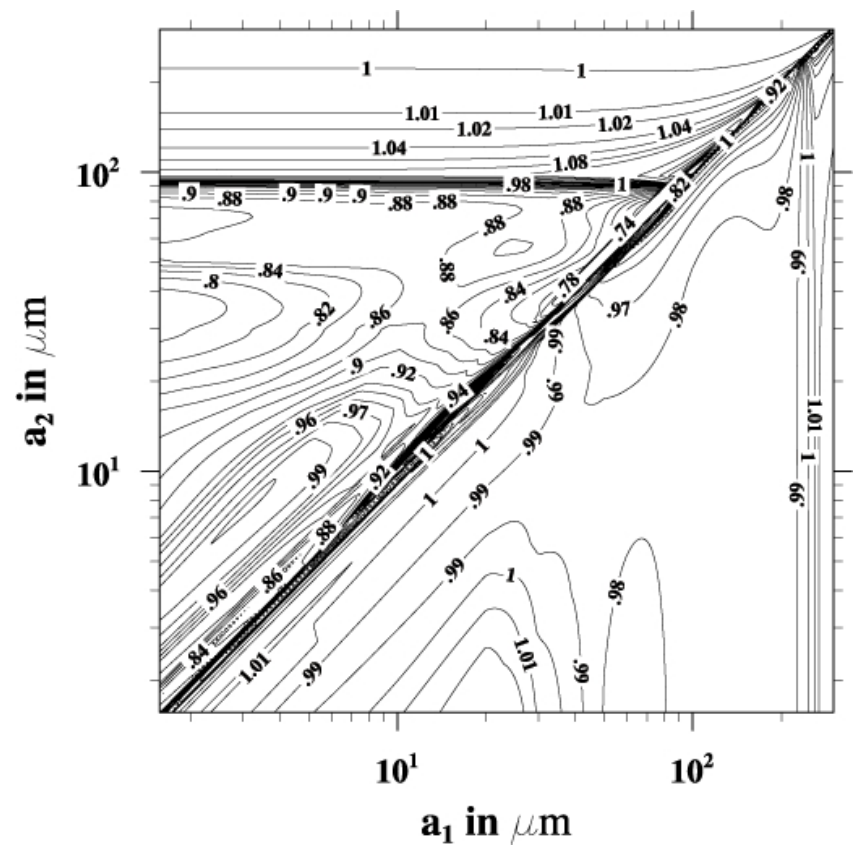

Fig. 1. Contour lines of the ratio $\mathcal{R}$ for $\epsilon_{2}=50 \mathrm{~cm}^{2} \mathrm{~s}^{-3}$ (lower right) and $\epsilon_{2}=500 \mathrm{~cm}^{2} \mathrm{~s}^{-3}$ (upper left), with $l_{2} / l_{1}=50$ and $l_{1}=$ $0.5 \mathrm{~m}$. Since $\mathcal{R}\left(a_{1}, a_{2}\right)=\mathcal{R}\left(a_{2}, a_{1}\right)$, therefore, only half of the domain is shown for each $\epsilon_{2}$.

Using the collection kernel formulation outlined in Sect. 2, we performed numerical integrations to obtain $\mathcal{R}$, for different droplet radii $a_{i}$ and $a_{j}$, the ratio $l_{2} / l_{1}$, and $\epsilon_{2}$. Figure 1 shows the contours of $\mathcal{R}$ for two typical dissipation rates and $l_{2} / l_{1}=50$. For most droplet size combinations, $\mathcal{R}$ is slightly less than one, implying that the dissipation intermittency reduces the effect of turbulence. Figure 2 shows $\mathcal{R}$ as a function of the flow dissipation rate for $l_{2} / l_{1}=10$ and 1000 for some typical droplet size combinations. In general, there is an increasing deviation from one as $\epsilon_{2}$ is increased. However, for small and intermediate dissipation rates, $\mathcal{R}$ does not deviate significantly from one (i.e., the difference is below $10 \%$ ).

Results highlighted by Figs. 2 and 1 can be understood as follows. Pair statistics relevant to collision-coalescence are second-order statistics and they tend to be dominated by regions of low flow dissipation rates which occupy most of the space. Increasing the intermittency implies a slight increase in the probability of the low dissipation-rate regions when the mean dissipation rate is fixed, and the higher dissipation rates take more extreme values. Furthermore, the extremedissipation regions occupy a small fraction of the volume, and their impact on the mean collection kernel is outweighed by the reduced kernel in low-dissipation regions.

Pinsky and Khain (2004) showed that a more realistic (i.e., non-Gaussian) PDF of flow accelerations actually reduced the value of radial relative velocity by 10 to $15 \%$ when compared to a Gaussian PDF. This level of reduction is quite similar to what is shown in Fig. 1 for $\epsilon_{2}=500 \mathrm{~cm}^{2} \mathrm{~s}^{-3}$. There

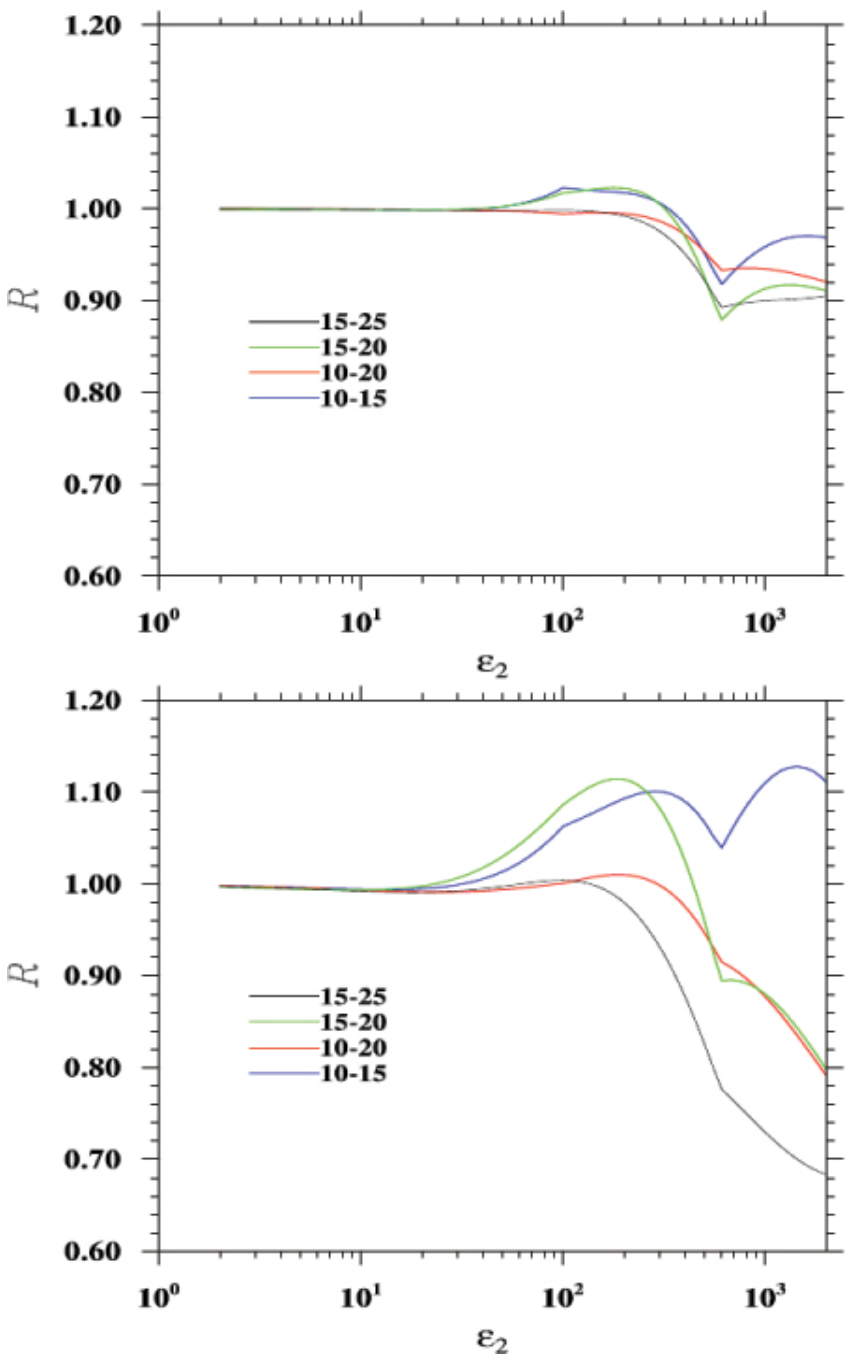

Fig. 2. The ratio $\mathcal{R}$ as a function of the dissipation rate $\epsilon_{2}$ (in $\mathrm{cm}^{2} \mathrm{~s}^{-3}$ ) for selected pairs of droplet radii (in $\mu \mathrm{m}$ ) and for $l_{2} / l_{1}=$ 10 (upper panel) and $l_{2} / l_{1}=1000$ (lower panel).

are also some droplet-size combinations where $\mathcal{R}$ is slightly larger than one, which could be a result of enhanced inertial clustering by intermittent flow events.

Flow intermittency is not the only effect when the DNS domain size and LES gridbox size differ significantly. Arguably, droplet spectra in DNS volumes with different dissipation rates will evolve differently; for instance, collision/coalescence will progress more rapidly in highdissipation rate volumes. Such effects are neglected in the above analysis because we consider enhancement of the kernel and not the enhancement of the spectral evolution in various DNS domains. In other words, the above analysis corresponds to the situation where the local DNS droplet spectra are always the same as the mean LES spectrum, which does not have to be the case. However, turbulence inside the LES gridbox also mixes droplets from various DNS domains, and the spectrum from a single DNS domain keeps its identity 
only for a limited time. The mixing progresses more rapidly for higher dissipation rates, that is, in cases where the role of turbulence intermittency becomes more significant according to the $\mathcal{R}$ analysis. Considering evolutions of the droplet spectra in various DNS domains rather than the $\mathcal{R}$ ratio exaggerates the impact unless some way of including the mixing between DNS domains is included in the analysis, an aspect beyond the scope of the current paper.

The above discussion, together with inherent limitations of the LES approach, suggests that the effect of dissipation intermittency due to the size gap between DNS and LES may be neglected, at least until more confident estimates of the turbulent enhancement are obtained and higher spatial resolution of bin-microphysics LES simulations becomes feasible.

\section{EULAG LES model with bin microphysics and effects of cloud turbulence}

\subsection{The model and setup of model simulations}

The LES model with bin microphysics is the same as in Wyszogrodzki et al. (2011; WGW11 hereafter) except that it is extended to include a representation of droplet growth by collision-coalescence. The fluid flow is calculated by the anelastic EULAG model (see Prusa et al., 2008 for a review and comprehensive list of references). The flow model is combined with the size-resolving representation of warmrain microphysics that includes droplet activation and growth by water vapor diffusion and by collision-coalescence, as described in Grabowski and Wang (2009) and Grabowski et al. (2011). The bin microphysics applies the linear flux method of Bott (1998) to calculate growth by collisioncoalescence. The number of bins in the microphysics scheme was selected as $\mathcal{N}=112$, which - together with the improved representation of the droplet activation - resulted in close-to-converged numerical solutions, as documented in Grabowski et al. (2011). The model also includes an additional predicted variable, the concentration of activated $\mathrm{CCN}$, which is needed when growth by the collision-coalescence is included. See Grabowski et al. (2011) for more details.

We consider simulations of a field of shallow cumuli based on the Barbados Oceanographic and Meteorological Experiment (BOMEX; Holland and Rasmusson, 1973) and used in the model intercomparison study described in Siebesma et al. (2003). EULAG application to the BOMEX case with either single-moment or double-moment bulk microphysics was reported in Grabowski (2006), Jarecka et al. (2009, 2011), Slawinska et al. (2012), and WGW11. Figure 3 presents initial zonal wind (meridional wind is assumed zero), temperature, and moisture profiles of the BOMEX setup. The profiles show the $1 \mathrm{~km}$-deep trade-wind convection layer overlaying the $0.5 \mathrm{~km}$-deep mixed layer near the ocean surface. The convection layer is covered by $0.5 \mathrm{~km}$ -
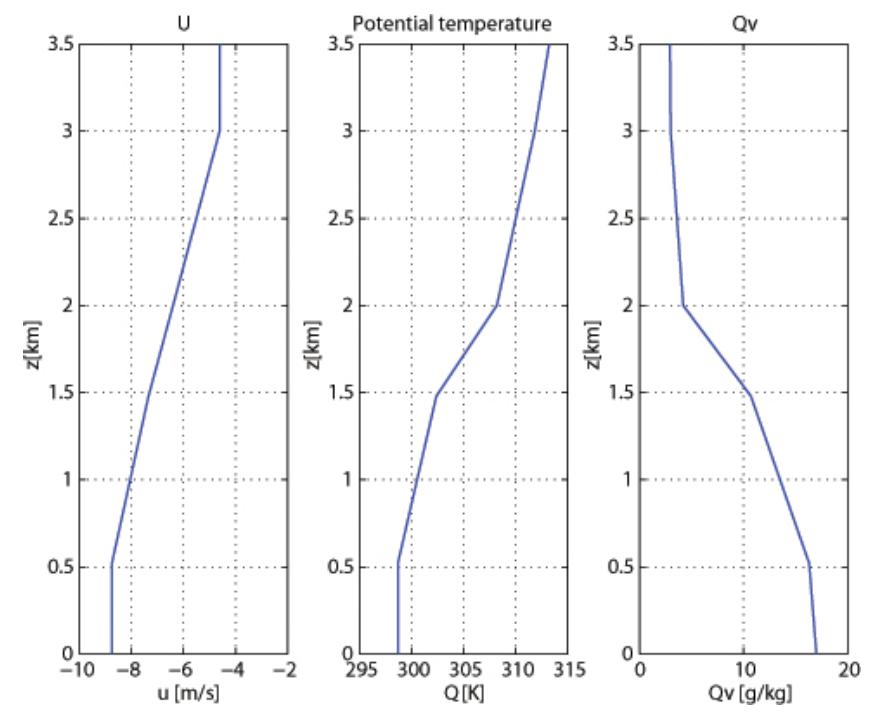

Fig. 3. Initial environmental profiles for the BOMEX simulation.

deep trade-wind inversion. The quasi-steady conditions are maintained by the prescribed large-scale subsidence, largescale moisture advection, surface heat fluxes, and radiative cooling. The original BOMEX case considers nonprecipitating convection, but we extend it here by adding precipitation processes. With precipitation, the steady-state conditions of the original BOMEX setup may be expected to evolve, but the mean temperature and moisture profiles at the end of all simulations are remarkably similar to those shown in Fig. 3 (not shown).

Details of the model setup are exactly as in WGW11, with horizontal/vertical gridlength of $\Delta x=\Delta y=50 \mathrm{~m}, \Delta z=$ $20 \mathrm{~m}$ and the domain size of $6.4 \mathrm{~km}$ in the horizontal and $3 \mathrm{~km}$ in the vertical. The model time step varies between 0.5 and $1.5 \mathrm{~s}$, depending on the strength of convection. The model is run for $6 \mathrm{~h}$ and two types of results are collected: (i) horizontally- and one-minute averaged statistics from the entire simulation for selected fields (e.g., cloud water mixing ratio, precipitation rate, etc.), and (ii) three-dimensional snapshots from the last $3 \mathrm{~h}$ collected every $5 \mathrm{~min}$.

Since the primary factor affecting the ability of an ice-free cloud to precipitate is the concentration of aerosol particles serving as $\mathrm{CCN}$, we consider a range of $\mathrm{CCN}$ concentrations by applying a general droplet activation formula in the form

$N_{\mathrm{CCN}}= \begin{cases}N_{\mathrm{CCN}}^{0} & \text { for } S>1 \\ N_{\mathrm{CCN}}^{0} S^{0.4} & \text { for } 0.1<S<1 \\ N_{\mathrm{CCN}}^{0}(0.1)^{-3.6} S^{4} & \text { for } S<0.1\end{cases}$

with $S$ in $\%$ and the total CCN concentration $N_{\mathrm{CCN}}^{0}$ of 30,60 , 120 , and $240 \mathrm{mg}^{-1}$. Such a range represents extremely clean to weakly polluted cloud conditions for subtropical shallow convective clouds. Simulations with the range of $\mathrm{CCN}$ concentrations will be referred to as $N 30, N 60, N 120$, and $N 240$. For each CCN conditions, three sets of simulations 


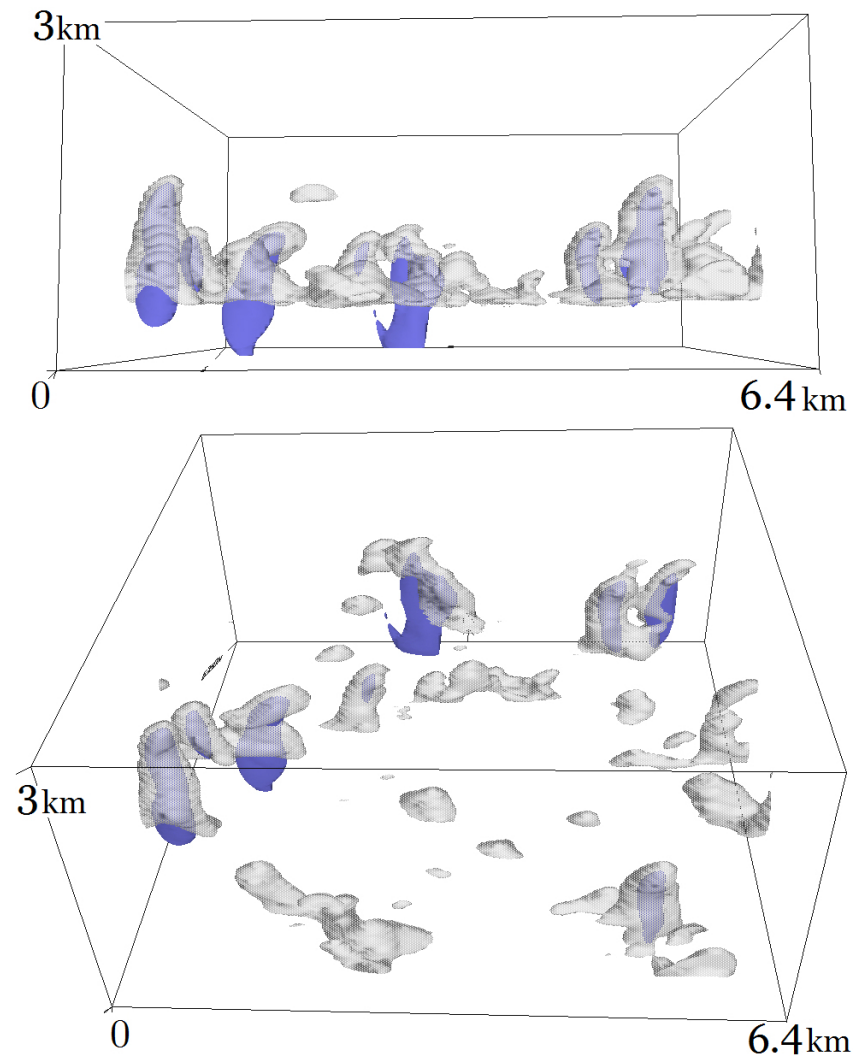

Fig. 4. Snapshots of cloud water mixing ratio $q_{\mathrm{c}}$ (transparent grey) and rain water mixing ratio $q_{\mathrm{r}}$ (solid blue) at the 6th hour of the simulation. The isosurfaces show values $q_{\mathrm{c}}=0.05 \mathrm{~g} \mathrm{~kg}^{-1}$ and $q_{\mathrm{r}}=$ $0.02 \mathrm{~g} \mathrm{~kg}^{-1}$.

were performed: (i) without collision-coalescence (i.e., as in WGW11), (ii) with collision-coalescence applying the gravitational collection kernel, and (iii) with collision-coalescence and turbulent enhancement of droplet collisions based on local characteristics of cloud turbulence.

As an example, Fig. 4 presents snapshots of the 3-D distribution of cloud fields at a time of $6 \mathrm{~h}$ for the $N 120$ case, marking cloudy volumes with cloud water mixing ratio $q_{\mathrm{c}}>0.05 \mathrm{~g} \mathrm{~kg}^{-1}$ with transparent grey color, and the rain water areas (blue color) for drizzle/rain mixing ratio $q_{\mathrm{r}}>$ $0.02 \mathrm{~g} \mathrm{~kg}^{-1}$. To calculate cloud and drizzle/rain water mixing ratios, the spectrum of drops is divided between the cloud and drizzle/rain assuming the drop threshold radius of $25 \mu \mathrm{m}$. We stress that the value of the threshold radius only affects the partitioning of the spectrum into cloud droplets and drizzle/rain drops (i.e., statistics of the results), and has no impact on the physics of the problem. The figure shows that several clouds, shallow and relatively deep, are present in the computational domain. They are at different stages of their life cycle, some precipitating and some not. In agreement with previous studies (e.g., Siebesma et al., 2003; Slawinska et al., 2012), the cloud coverage is typically around $10 \%$.

\subsection{Turbulent enhancement of the collection kernel in LES model}

Because of relatively coarse resolution of the LES simulations, turbulent processes within simulated clouds remain mostly parameterized. In particular, the model solves a prognostic equation for the turbulent kinetic energy (TKE), as described in Margolin et al. (1999), with parameters adopted from Schumann (1991). The grid-volume averaged dissipation rate $\epsilon$ is derived from TKE as

$\epsilon=C_{\epsilon}(\mathrm{TKE})^{3 / 2} / \Delta$

where $\Delta=(\Delta x+\Delta y+\Delta z) / 3$ is the arithmetic average of model grid increments, and $C_{\epsilon}=0.845$. The flow Reynolds number $R e_{\lambda}$ is defined using the root mean square (rms) velocity $u^{\prime}$ as (Pope, 2000; Wang et al., 2006a)

$R e_{\lambda}=15^{1 / 2}\left(u^{\prime} / v_{\mathrm{K}}\right)^{2}$

where $v_{\mathrm{K}} \sim \epsilon^{1 / 4}$ is the Kolmogorov velocity. The rms velocity is derived assuming that $\epsilon \sim u^{\prime 3}$ (Pope, 2000) and selecting $u^{\prime}=2.02 \mathrm{~m} \mathrm{~s}^{-1}$ for $\epsilon=400 \mathrm{~cm}^{2} s^{-3}$ (Wang et al., 2006a). This gives an empirical formula for $u^{\prime}\left(\right.$ in m s$\left.^{-1}\right)$ in the form

$u^{\prime}=2.02 \cdot(\epsilon / 400)^{1 / 3}$

with $\epsilon$ expressed in $\mathrm{cm}^{2} \mathrm{~s}^{-3}$. Note that Eqs. (11) and (12) imply $R e_{\lambda} \sim \epsilon^{1 / 6}$. For small droplets (say, radius smaller than $30 \mu \mathrm{m}$ ) the collection kernel is not affected by $u^{\prime}$, while for larger droplets $u^{\prime}$ (or $R e_{\lambda}$ ) has a secondary effect on the kernel. Overall, the increase of TKE (and thus $\epsilon, u^{\prime}$, and $R e_{\lambda}$ ) shortens the time needed to form drizzle drops. The growth times also depend on the liquid water content (LWC), but the relative reduction of the growth time for a given dissipation rate is similar for different LWCs due to the self-similarity of the kinetic collection equation.

\section{Results}

\subsection{Preamble: rising thermal simulations}

We start with a brief discussion of results of more idealized (2-D single-cloud) simulations that help better explain effects of turbulence on warm-rain formation in realistic cloudfield simulations. We apply the idealized model setup previously considered in Grabowski et al. (2010; Sect. 5). In this setup, an initially circular moist warm thermal (a bubble) rises in the stratified environment and forms a cloud. As cloud droplets grow by the diffusion of water vapor and eventually by collision-coalescence, drizzle and rain develop, with rain falling out of the cloud and reaching the surface. Removal of cloud water from the cloud and mixing with subsaturated cloud environment leads to cloud dissipation and rain cessation. 

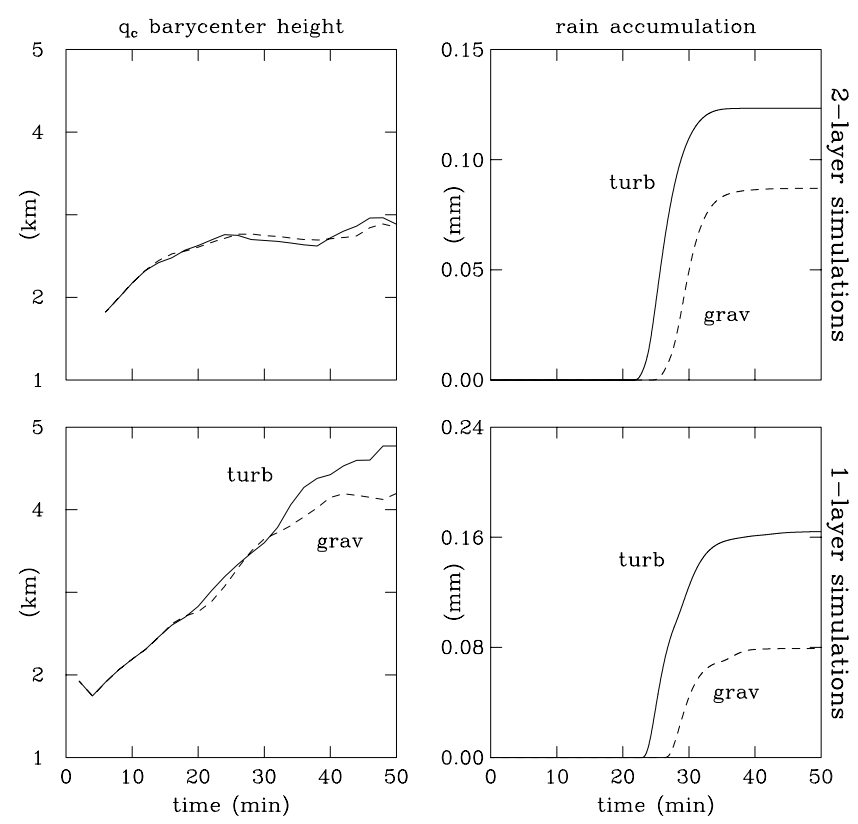

Fig. 5. The evolution of the height of the cloud water center of mass (left panels) and the mean (horizontally-averaged) surface rain accumulation (right panels) for 2-D rising thermal simulations. Upper panels come from simulations where the thermal rise is arrested in the middle of the domain by the layer of increased stability. Lower panels show results from simulations where the thermal can rise unobstructed towards the upper model boundary.

For reasons that will become obvious while discussing the results, we consider two model setups featuring different environmental conditions. The first setup (upper panels in Fig. 5) is exactly as in Grabowski et al. (2010) and features two layers in two halves of the domain vertical extent. The lower/upper layer features static stability of $1.3 / 3.0 \times$ $10^{-5} \mathrm{~m}^{-1}$. In this setup, the initial perturbation rises across the lower layer, but its further vertical development is arrested by the presence of the more stable upper layer (see Fig. 8 in Grabowski et al., 2010). The second setup (lower panels on Fig. 5) assumes a single-layer atmospheric structure, with the static stability of $1.6 \times 10^{-5} \mathrm{~m}^{-1}$ and relative humidity of $80 \%$. An initial perturbation, the same as in Grabowski et al. (2010), rises across the domain and reaches levels close to the upper model boundary (at $5 \mathrm{~km}$ ) near the end of these simulations. Two simulations are performed for each model setup applying EULAG with the same bin microphysics scheme as in cloud field simulations. The first simulation applies the gravitational collection kernel. The second simulation includes effects of cloud turbulence in an extremely simplified way, namely, by assuming that the turbulence intensity at all cloudy points corresponds to a constant eddy dissipation rate of $\epsilon=100 \mathrm{~cm}^{2} \mathrm{~s}^{-3} \cdot N_{\mathrm{CCN}}^{0}$ of $120 \mathrm{mg}^{-1}$ is assumed in Eq. (9) for all four simulations.

A synthesis of model results is presented in Fig. 5. The figure shows evolution of the height of the cloud water center of mass (the barycenter) and the total accumulated surface precipitation. As expected, thermals in simulations with the inversion rise to approximately similar height as documented by the barycenter height evolutions. The key result is that rain reaches the surface about 4 min earlier in the turbulent case, and there is about $40 \%$ more total accumulated rain in this case. Arguably, the latter comes from earlier (in the cloud life cycle) formation of initial drizzle drops from cloud droplets through the autoconversion phase of rain formation. This gives more cloud water available throughout the rest of the cloud life cycle for the accretion phase of the rain development, when existing drizzle and rain drops collect cloud droplets.

When thermals are allowed to rise without the inversion, the difference in the total accumulated rain is even larger, a factor of two. It means that the "microphysical enhancement" (i.e., earlier formation of initial drizzle through the autoconversion phase) is supplemented by an additional mechanism. As the differences in the height of the LWC center of mass suggest, the thermal applying turbulent kernel rises to higher levels and provides more condensed water for the rain formation. Arguably, the difference comes from "off-loading" the condensed water through rain formation, which is more effective when the turbulent kernel is used. Condensed water reduces cloud positive buoyancy and its removal leads to increased buoyancy and thus a possibility of reaching higher levels. This "dynamical enhancement" adds to the microphysical enhancement discussed above and they both result in an astonishing overall effect evident in the lower panels.

One should treat the above results with significant caution. For instance, another aspect of these simulations is that thermals rising without the inversion actually produce less rain at the surface than thermals impinging upon the inversion (compare scales on vertical axes in the right panels). This may be because more rain evaporates during its fall from the cloud to the surface when thermals reach higher levels. Assumed 2-D geometry, simple cloud forcing (a bubble), and constant in space and time characteristics of the cloud turbulence all make the model results discussed above qualitative. Nevertheless, the results highlight mechanisms involved, the significance of combined microphysical and dynamical factors in particular. These will play an important role in cloud field simulations presented in subsequent sections.

\subsection{Statistics of cloud properties in cloud field simulations}

Figure 6 presents contoured frequency by altitude diagrams (CFADs; the probability density function calculated at each height and applying colors to mark the frequency of occurrence at various heights) of the dissipation rate inside cloudy volumes (left panel), and the adiabatic fraction $\mathrm{AF}$ (the ratio between the local LWC and the adiabatic LWC at a given height, the latter estimated from the simulated mean cloud 

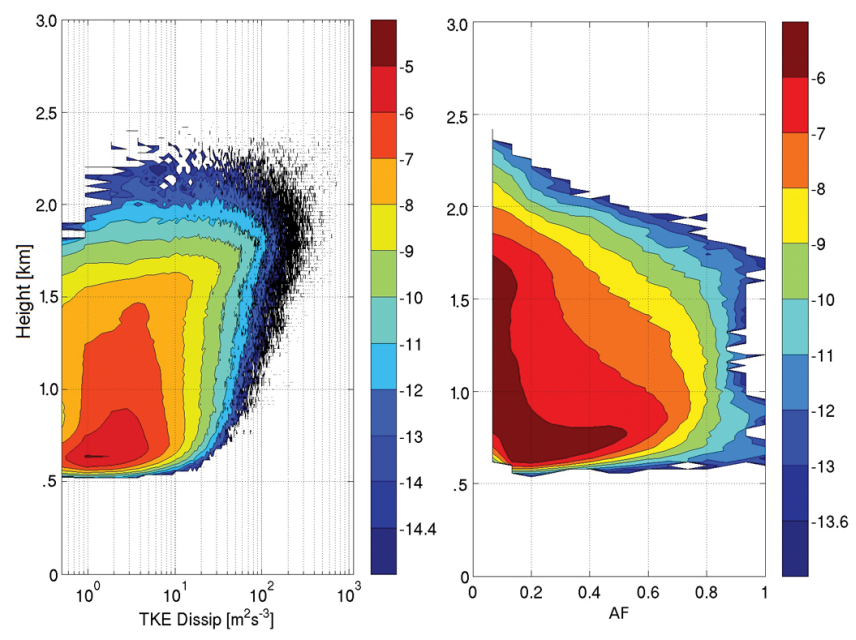

Fig. 6. The contoured frequency by altitude diagrams (CFADs) of the dissipation rate (in $\mathrm{cm}^{2} \mathrm{~s}^{-3}$ ) and adiabatic fraction AF inside volumes with cloud water mixing ratio $q_{\mathrm{c}}>0.01 \mathrm{~kg} \mathrm{~kg}^{-1}$. The logarithm of the frequency (with respect to the number of cloudy points at a given height) is indicated by the color bars.

base height and the initial sounding; right panel) for the $N 120$ simulation. Plots for other simulations are similar (not shown). The figure documents features well established from numerous observations and simulations of shallow convection: large variability of local cloud characteristics at a given height, significant cloud dilution with the mean AF decreasing with height, turbulence intensity with typical $\epsilon$ values between a fraction of $1 \mathrm{~cm}^{2} \mathrm{~s}^{-3}$ and a few tens $\mathrm{cm}^{2} \mathrm{~s}^{-3}$, and the maximum $\epsilon$ values reaching several hundreds $\mathrm{cm}^{2} \mathrm{~s}^{-3}$ in the upper part of the cloud field. One might anticipate formation of initial drizzle drops in volumes featuring high $\mathrm{AF}$ and an acceleration of this process if significant levels of cloud turbulence are present. Inspection of cloud field snapshots documents that the highest turbulence intensity typically occurs near cloud edges in upper parts of individual cumuli, and this is where the highest LWC is often found. Such a picture agrees with the discussion in Seifert et al., 2010 (see their Fig. 6 in particular).

Figure 7 shows percentile distributions of the cloud droplet concentration for the N120 with and without collisioncoalescence. Results of the simulation without collisioncoalescence are similar to those shown in WGW11: distributions are relatively wide with the most frequent concentration values approximately constant with height (except in the uppermost $0.5 \mathrm{~km}$ of the cloud field depth). As documented in Slawinska et al. (2012) and WGW11, the almost constant mean droplet concentration as a function of height (also observed in field studies; see discussion in Slawinska et al., 2012) comes from the secondary (i.e., above the cloud base) activation of CCN. Distributions for simulations with collision-coalescence are similar to those without collisions, except that clouds with turbulent collisions appear reaching

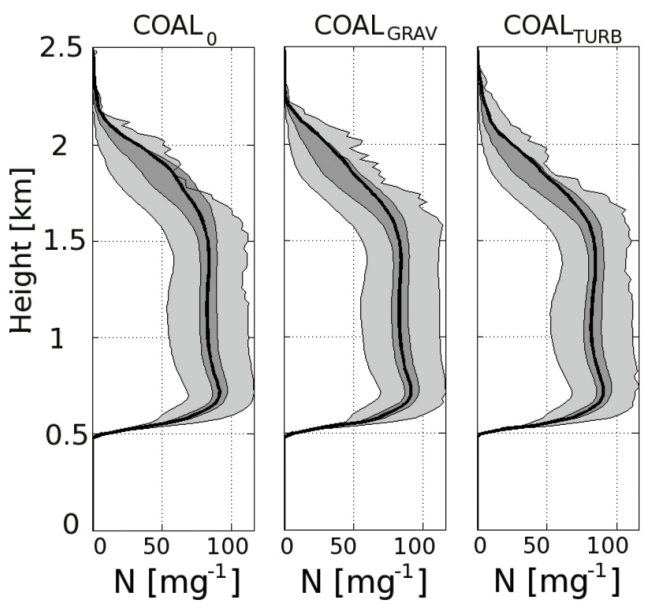

Fig. 7. Percentiles of the total drop concentration for the $N 120$ case. Left, middle, and right panels show results from simulations without collision-coalescence, with gravitational collision-coalescence, and with turbulence-enhanced collision-coalescence, respectively.

higher levels. Similar distributions are true for other simulations (see Figs. 11 and 15 to be discussed later). Overall, the presence of precipitation seems to have only a small impact on CFADs of the droplet concentration.

Figure 8 shows CFADs of the effective radius, the ratio between the third and the second moment of the entire drop size distribution, for the $N 120$ case. Effective radius, together with the local LWC, determines local cloud properties relevant to the transfer of the solar radiation, and it is typically slightly larger than the mean volume radius (i.e., the radius of the mean mass droplet, the LWC divided by the droplet concentration). The figure also shows profiles of the effective radius for adiabatic monodisperse spectra of cloud droplets corresponding to droplet concentrations of 90, 120, and $150 \mathrm{mg}^{-1}$. Without collision-coalescence, CFAD of the effective radius is relatively wide, and the maximum frequency is consistent with the adiabatic profiles. With gravitational collision-coalescence, the maximum frequency begins to shift to the right (i.e., toward larger sizes) in the upper part of the cloud field. This is barely visible for the gravitational coalescence but becomes obvious when the effects of turbulence are included. For simulations with lower CCN, the shift towards larger droplet sizes is apparent even with the gravitational coalescence, but it is barely visible for turbulenceenhanced collisions with the highest $\mathrm{CCN}$ concentration considered (not shown).

\subsection{Rainfall in cloud field simulations}

Figures 9 and 10 show evolutions of the horizontallyaveraged cloud water and the drizzle/rain water mixing ratios, respectively, applying again the $25 \mu \mathrm{m}$ threshold to locally partition the droplet/drop spectrum into cloud and drizzle/rain. The Fig. 9 panels show that the presence of 


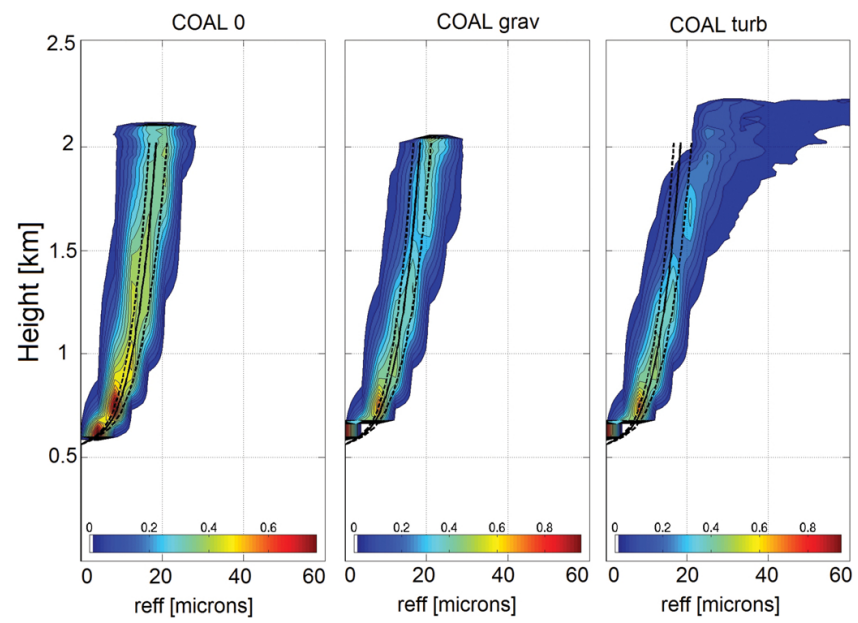

Fig. 8. CFAD of the effective radius $\left(r_{\text {eff }}\right)$. Solid and dashed lines represent the adiabatic model profiles for the $\mathrm{CCN}$ concentration of $120 \mathrm{mg}^{-1}$ and $90 / 150 \mathrm{mg}^{-1}$.

significant drizzle/rain strongly affects mean cloud water profiles in both gravitational and turbulent cases. In low $\mathrm{CCN}$ simulations $N 30$ and $N 60$, cloud water is clearly reduced in the upper part of the cloud field due to its efficient removal by drizzle/rain. The figure also shows oscillations of the cloud field due to interactions between clouds and their environment as well as the random nature of cloud initiation and evolution. Mean cloud water above the cloud base features periods of enhanced horizontally-averaged cloud water that develop from the cloud base upwards as tilted yellow streaks with the highest mean cloud water (red color) at some of their tops, especially in high-CCN simulations. These oscillations of the mean cloud field are also apparent in the time evolution of the horizontally-averaged drizzle/rain water mixing ratio shown in Fig. 10, with the highest mean drizzle/rain in the upper parts of the cloud field and in periods of enhanced mean cloud water. The amount of drizzle/rain strongly increases with the decrease of $\mathrm{CCN}$ concentrations (note different color scales in panels corresponding to various $\mathrm{CCN}$ concentrations) as one might expect. Overall, the mean drizzle/rain seems higher when the turbulent kernel is used, an aspect quantified in the subsequent analysis. Enhanced drizzle/rain in the upper parts of the cloud field (red colors) typically corresponds to periods with deeper clouds and provides initiation points of downward streaks as enhanced rain falls towards the ocean surface. Overall, Figs. 9 and 10 clearly demonstrate that the impact of the turbulent kernel on the macrophysical cloud field properties seems less significant than the impact of the assumed CCN concentrations.

Figure 11 shows evolutions of the precipitation fraction profiles for all eight simulations. Precipitation fraction is an analog of the cloud fraction, that is, the fraction of the horizontal area covered by clouds at a given height. At each height, precipitation fraction is defined as the fraction of the horizontal domain with drizzle/rain flux larger than $3.65 \times$ $10^{-5} \mathrm{~kg} \mathrm{~kg}^{-1} \mathrm{~m} \mathrm{~s}^{-1}$. The specific threshold comes from the estimated cutoff precipitation flux of the precipitation radar; see Sect. 2.4.2 in Van Zanten et al. (2010). Because of the fluctuations of cloud and precipitation fields, 1 min precipitation fraction profiles vary significantly. The average profiles, on the other hand, clearly illustrate differences between various simulations, the impact of $\mathrm{CCN}$ and turbulent enhancement in particular. In simulations $N 240$ (bottom row), precipitation is only present in the upper parts of the cloud field (consistent with Fig. 10), with some downward extension of the mean profile in the turbulent kernel case. In the $N 120$ case, precipitation seems to reach the surface only when turbulent collection kernel is considered. In N60 and $N 30$ cases, a significant increase of the precipitation fraction (factor of two) is simulated with turbulent collisions. It is also apparent that profiles corresponding to the turbulent kernel feature a deeper cloud field, with profiles for the turbulent kernel approaching zero at heights around or above $2.5 \mathrm{~km}$, whereas profiles for the gravitational kernel terminate between 2.2 and $2.3 \mathrm{~km}$. This represents the dynamic enhancement, that is, a more efficient off-loading of cloud condensate in the case of turbulent kernel. Instead of the precipitation fraction, one may consider profiles of the precipitation flux because its time-averaged surface value represents surface rain accumulation. Such profiles provide a similar message as Fig. 11 and are not shown.

Figure 12 presents the time evolution of the domainaveraged cloud water path (CWP) and precipitation water path (PWP), namely, vertical integrals of the mean cloud water and drizzle/rain water contents, respectively, for simulations with gravitational and turbulent kernels. The figure represents a more comprehensive representation of model results shown in Figs. 9 and 10. CWP (as well as PWP in simulations with significant rain) fluctuates significantly as cloud fields evolve. Simulations N240 and N120 show similar mean CWP values and little PWP, in agreement with the previous discussion. In contrast, simulations $N 60$ and $N 30$ show significant differences between mean PWP and approximately the same CWP. The increased mean PWP in turbulent $N 60$ and $N 30$ cases represents effects of turbulence on drizzle/rain formation. Enhanced drizzle/rain implies more efficient removal of cloud water, as illustrated by the differences in low and high CCN simulations in Fig. 9. It follows that the only explanation why the CWP remains approximately the same in corresponding turbulent and gravitational simulations (around 11.2 and $7.1 \mathrm{~g} \mathrm{~m}^{-2}$ in $N 60$ and $N 30$, respectively) is the dynamic enhancement, that is, occasional presence of deeper clouds in simulations applying turbulent kernel.

To further quantify the dynamical enhancement, distributions of the cloud top height for all simulations were obtained from snapshots of the cloud fields for hours 3 to 6 . The cloud top was defined as the height at which the sum of the CWP and PWP (integrated from the model top downwards) 

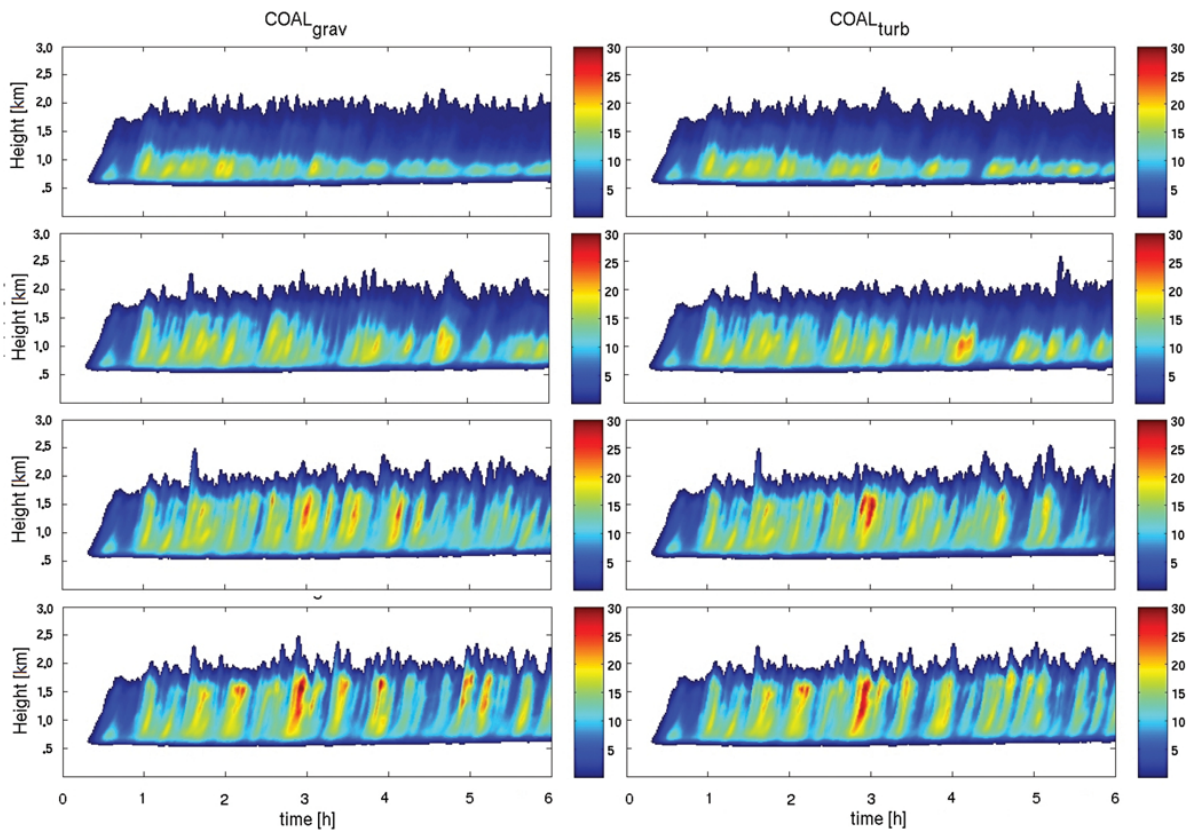

Fig. 9. Time evolution of the horizontally averaged cloud water mixing ratio. Left (right) column shows cases with the gravitational (turbulence-enhanced) kernel. Rows from top to bottom are for N30, N60,N120, and N240, respectively. Color scale depicts units of $\mathrm{mg} \mathrm{kg}^{-1}$.
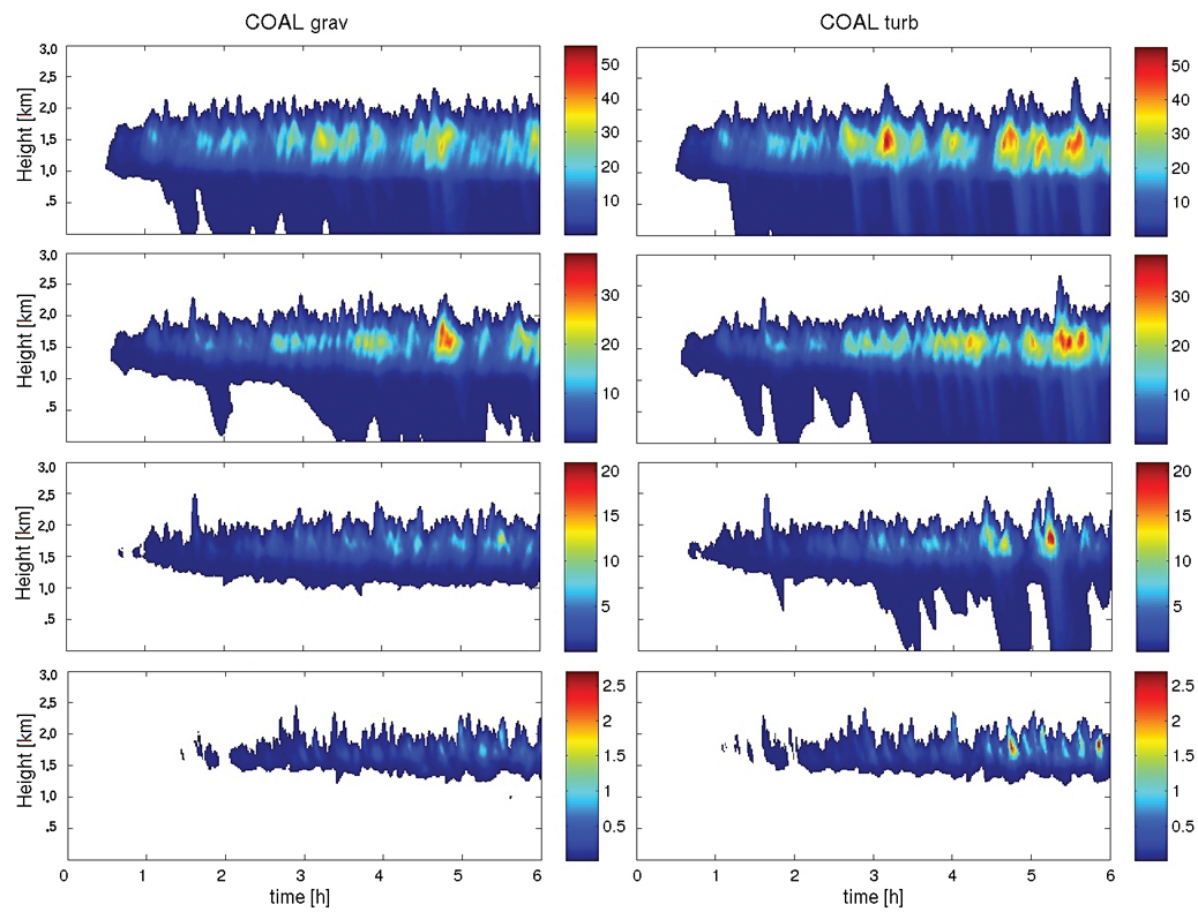

Fig. 10. As in Fig. 9 but for above but for the precipitating (drizzle/rain) water mixing ratio. Color scale depicts units of $\mathrm{mg} \mathrm{kg}^{-1}$.

reaches $10 \mathrm{~g} \mathrm{~m}^{-2}$. Figure 13 compares normalized distributions (i.e., the probability density functions; pdfs) for the simulations $N 30$ and N240 and with the gravitational and turbulent kernels. The figure shows that distributions are bimodal, with one peak for cloudy columns reaching height of about $750 \mathrm{~m}$ (i.e., less than $200 \mathrm{~m}$ above the cloud base) and the second peak around $1500 \mathrm{~m}$. For the non-precipitating N240 cases, distributions for gravitational and turbulent kernels 

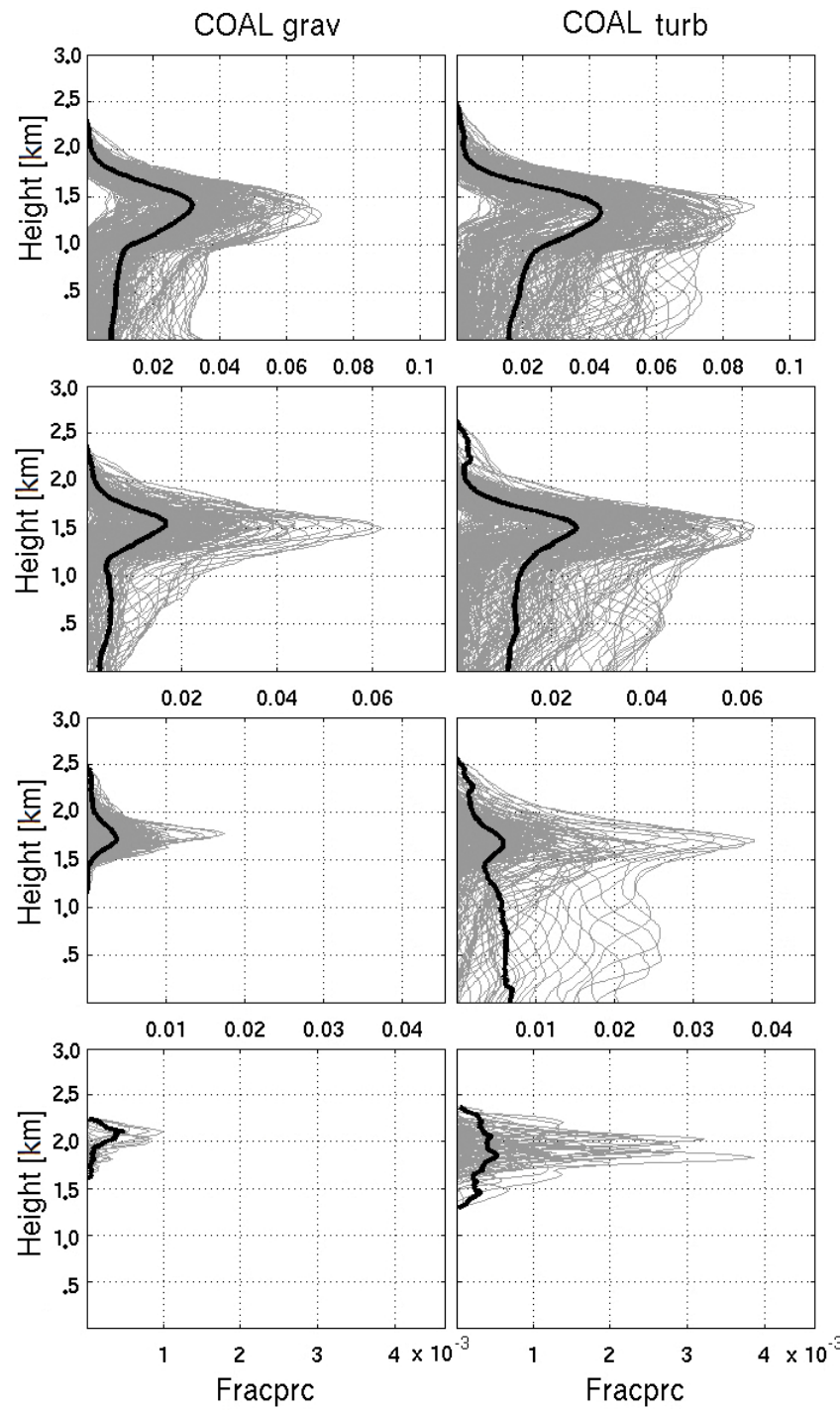

Fig. 11. Profiles of the precipitation fraction (Fracprc) for simulations with (left column) gravitational kernel and (right column) turbulent kernel, and for simulations (top to bottom) N30, N60, N120, and $N 240$. Grey lines represent evolution of 1 min average profiles between hours 1 and 6; black thick line is the average of the grey profiles.

are almost identical, with the $750 \mathrm{~m}$ peak significantly larger than the $1.5 \mathrm{~km}$ peak. Precipitating $N 30$ case features distributions with the $1.5 \mathrm{~km}$ peak larger than the $750 \mathrm{~m}$ peak, suggesting significant differences in the cloud field when shallow convective clouds heavily precipitate. The change in magnitude of the two peaks is further accentuated when turbulent kernel is used. Other simulations feature distributions between the two extremes shown in the figure (not shown). Differences between simulations applying gravitational and turbulent kernels for the $N 30$ case clearly illustrate macroscopic changes of the cloud field due to the dynamical enhancement.

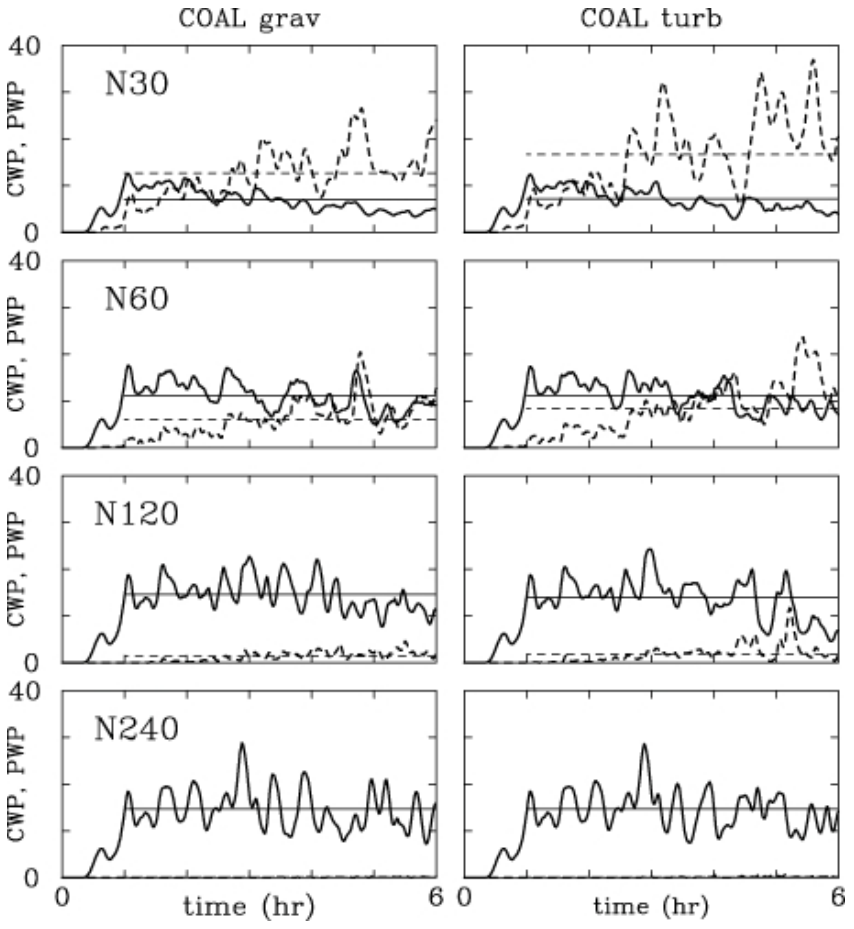

Fig. 12. Evolution of the 1 min domain-averaged CWP (thick solid lines) and PWP (thick dashed lines) for the gravitational (left panels) and turbulent (right panels) kernel simulations $N 30, N 60$, $N 120$, and N240, from top to bottom, respectively. CWP and PWP is in $\mathrm{g} \mathrm{m}^{-2}$. The thin solid/dashed lines show $5 \mathrm{~h}$ average CWP/PWP between hours 1 and 6 of the simulation.

Figures 14 and 15 show evolutions of the cumulative rainfall at the cloud base and at the surface (i.e., integrated over time mean precipitation flux at these heights) in simulations $N 30, N 60$, and $N 120$ applying linear and logarithmic scales, respectively. The linear plot clearly shows the enhancement factor between simulations applying gravitational and turbulent kernel, whereas the logarithmic plot allows comparison between lower and higher precipitation cases. We consider both the cloud base and the surface to document effects of rain evaporation between the cloud layer and the surface, but this aspect is only marginally relevant, as shown by the figures. Horizontally-averaged surface rain accumulations over the last $3 \mathrm{~h}$ of the gravitational kernel simulations are 0.013 and $0.001 \mathrm{~mm}$ for $N 30$ and $N 60$, respectively. For the turbulent kernel, the accumulations are 0.045 (N30) and $0.013 \mathrm{~mm}(N 60)$. When averaged only over areas with nonvanishing surface precipitation (thus taking into account a small cloud fraction and even smaller fraction of precipitating clouds; see Fig. 11), the $3 \mathrm{~h}$ accumulations for gravitational/turbulent kernel simulations are $0.80 / 1.47 \mathrm{~mm}$ for $N 30$ and $0.11 / 0.66 \mathrm{~mm}$ for $N 60$. The key point is that regardless whether the cloud base or the surface is considered, or whether the entire domain or only precipitating areas are included, the turbulent enhancement of droplet collisions has 

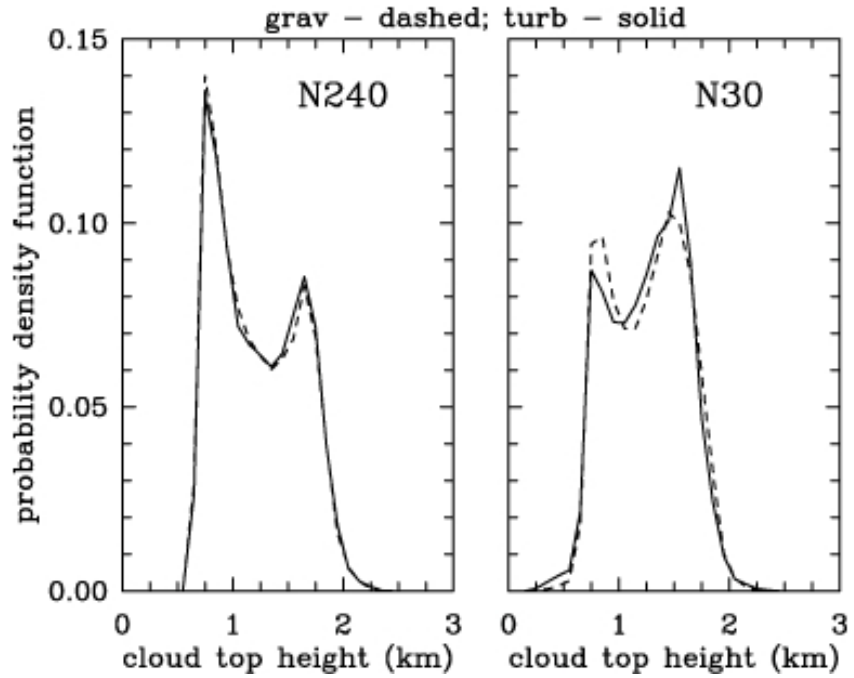

Fig. 13. Probability density function of the cloud top height: simulation $N 240$ (left panel) and $N 30$ (right panel); the gravitational and turbulent kernels are denoted by dashed and continuous lines, respectively.
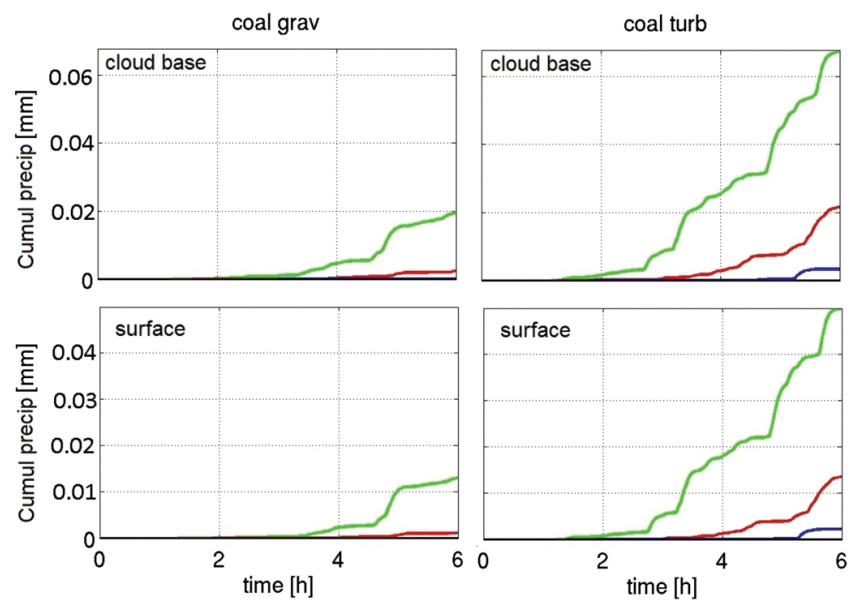

Fig. 14. Evolution of the cumulative precipitation (cumul precip) flux [mm] at the cloud base (upper panels) and at the surface (bottom panels) for gravitational (left) and turbulent (right) collection kernels. Green, red and blue lines represent evolutions for $N 30$, $N 60$, and $N 120$ simulations, respectively.

a strong impact, with rain accumulations several times larger than applying the gravitational kernel. Such an effect qualitatively agrees with the impacts reported in Seifert et al., 2010 (see their Table II in particular).

\section{Summary and conclusions}

This paper discusses LES simulations of a field of shallow convective clouds aiming at quantitative assessment of the impact of cloud turbulence on warm rain processes. The
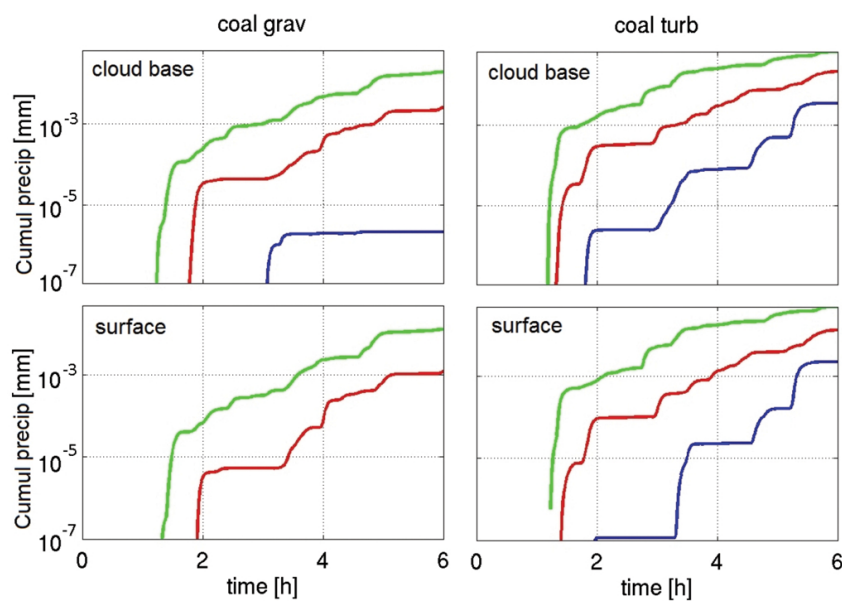

Fig. 15. Same as Fig. 14 but with the logarithmic scale on the vertical axis.

study provides a climax of around a decade long collaborative research between the University of Delaware and the NCAR (National Center for Atmospheric Research, Colorado, USA) that resulted in the development of cloud droplet collection kernels, including effects of cloud turbulence. These kernels were developed through theoretical studies guided by DNS and more recently HDNS simulations. Effects of turbulence on rain formation is a difficult multiscale problem, concerning processes taking place over a wide range of spatial scales. Formation and growth of cloud droplets (by water vapor diffusion and collision-coalescence) take place at scales from submicron to tens and hundreds of microns. Small-scale cloud dynamics concern scales within the inertial range of atmospheric turbulence, from scales at which cloud TKE is generated (tens to hundreds of meters) down to the Kolmogorov microscale, around a millimeter in typical atmospheric conditions. Larger-scale cloud and precipitation dynamics involve such processes as cloud initiation, formation of cloud updrafts and downdrafts, interactions between precipitation-laden downdrafts and the surface, etc. They involve spatial scales of hundred of meters to a few kilometers. Finally, at even larger scales, mesoscale processes determine overall characteristics of a field of precipitating clouds (cloud depth, cloud cover, etc.). The range, from submicron to tens of kilometers, represents about 10 decades of spatial scales and it will never be resolved in the numerical simulation. However, with the availability of the petascale computing resources, we aim at extending our collaborative research towards reducing the scale gap between top-down LES of cloud dynamics (bin EULAG) and a bottom-up HDNS of cloud microphysics. Ultimately, both approaches may result in the near future in an integrated multiscale (in time and space) simulation environment, with the two approaches overlapping at the $O(1 \mathrm{~m})$ spatial scales. This effort will offer an opportunity to develop 
new parameterizations of various cloud physical processes unresolved in weather and climate models.

We applied previously developed collection kernels that include effects of cloud turbulence to LES cloud-scale simulations using bin microphysics and targeting shallow convective clouds where turbulence effects are expected to be significant. Except for applying the bin microphysics, our study is similar to Seifert et al. (2010), in which the simulation setup based on the RICO (Rain In Cumulus over Ocean) model intercomparison case was used (Van Zanten et al., 2010). We apply the BOMEX case (Siebesma et al., 2003) because it maintains the initial atmospheric state in the nonprecipitating case, and it was used in our previous studies (e.g., Slawinska et al., 2012; Wyszogrodzki et al., 2011). As documented in Stevens and Seifert (2008) and Seifert et al. (2010), RICO simulations feature a gradual deepening of the cloud field (because of the increase of the inversion height) and consequently a gradual increase of the surface precipitation rate. Since the rate at which the cloud field deepens seems to depend on the water budget within the upper part of the cloud field (and the budget is affected by precipitation processes, Stevens and Seifert, 2008), the RICO setup involves additional complications (e.g., feedbacks) that we avoid by applying the BOMEX setup.

Before applying the turbulent kernel in LES simulations, we first addressed the role of the flow intermittency, an aspect not considered in previous studies. This is a relevant issue and its importance can be justified in the following way. The turbulent enhancement of the gravitational kernel depends nonlinearly on the turbulence characteristics, and these characteristics strongly fluctuate in time and space due to the flow intermittency. Because of computational limitations, the LES gridbox is typically much larger than the computational domain applied in the DNS and HDNS studies. It follows that the mean dissipation rate predicted by LES may represent rate of droplet collisions that is different from the rate that considers spatial variability of the dissipation rate. We investigated this problem by comparing the turbulent kernel derived applying the mean dissipation rate with the averaged kernel applying the distribution of the dissipation rates, following the Kolmogorov (1962) refined similarity theory. The analysis showed, perhaps to some surprise, that the effects become significant (the relative difference above 10\%) only for high dissipation rates (above $100 \mathrm{~cm}^{2} \mathrm{~s}^{-3}$ ) and large (higher than 10) ratios between LES grid length and DNS domain size. As a result, and considering still uncertain formulation of the turbulent kernel, we decided to exclude these effects from our analysis. Consequently, we simply apply the turbulence-enhanced collection kernels based on the local TKE predicted by the LES model and exclude effects of the subgrid-scale variability of TKE dissipation. One also need to keep in mind that such a formulation excludes effects of the delay between TKE generation at scales close to LES grid length (e.g., through the interfacial instabilities, cf. Grabowski and Clark, 1991, 1993) and the TKE dissipa- tion when TKE reaches the Kolmogorov microscale. Such a delay can be significant (tens of seconds; see section 5 in Grabowski and Clark, 1993) and lead to additional uncertainty. All these issues become less problematic once the LES gridlength approaches the size of the computational domain applied in DNS and HDNS studies.

To highlight physical processes responsible for the impact of cloud turbulence on the rain development, we presented simple 2-D simulations of a precipitating thermal. These simulations show that rain develops earlier and more rain falls from the thermal when turbulent effects are included. The former effect was anticipated based on our previous idealized studies and it comes from a more rapid autoconversion phase of rain formation. The latter effect comes from a combination of two different mechanisms. Firstly, if drizzle forms earlier, then more cloud water is available to be converted into precipitation throughout the cloud life cycle. We refer to this as the microphysical enhancement. Secondly, when rain develops and falls from a cloudy volume, then the buoyancy of the volume is increased and the volume can rise higher and produce more cloud water to be converted into precipitation. This feedback from the cloud microphysics into cloud dynamics can be referred to as the dynamical enhancement. In idealized rising thermal simulations, the microphysical and dynamical enhancement contribute about equally to the overall effect, with simulations including turbulent effects resulting in about a twofold increase of the surface rainfall. However, these simulations need to be treated with much caution because of their significant simplifications.

Cloud field simulations also show a combination of microphysical and dynamical enhancements, although quantification of their relative contribution is more difficult. Because the primary factor affecting ability of a cloud to precipitate is the concentration of cloud droplets (which determines the maximum size of diffusionally grown droplets given the cloud depth), we performed simulations with a range of prescribed CCN concentrations $\left(30,60,120\right.$, and $\left.240 \mathrm{mg}^{-1}\right)$. Only small amounts of drizzle/rain were simulated within clouds for the highest $\mathrm{CCN}$ concentration, and the turbulent kernel led to increased amounts of drizzle/rain. Rain below the cloud base was present neither in gravitational nor in turbulent kernel simulations. For the $\mathrm{CCN}$ concentration of $120 \mathrm{mg}^{-1}$, rain occasionally reached the surface in the turbulent kernel simulation, but not when applying the gravitational kernel. In simulations using 30 and $60 \mathrm{mg}^{-1}$ $\mathrm{CCN}$ concentrations, rain reached the surface regardless of the type of kernel used. However, the $6 \mathrm{~h}$ accumulations were significantly larger when using the turbulent kernel. The domain-averaged surface accumulations were around 0.013 and $0.001 \mathrm{~mm}$ for the gravitational kernel assuming 30 and $60 \mathrm{mg}^{-1} \mathrm{CCN}$ concentration, respectively, and around 0.050 and $0.013 \mathrm{~mm}$ for corresponding simulations applying turbulent kernel. These imply a dramatic increase when effects of cloud turbulence are included, in line with the enhancement presented in Seifert et al., 2010 (see their Table II therein). 
The dynamical enhancement results in a larger contribution of deeper clouds to the entire cloud population, and leads to similar time-averaged CWPs between gravitational and turbulent kernel simulations despite significant differences in PWP and rainfall. Simulations with small or no rainfall below the cloud base showed only effects of the microphysical enhancement.

Simulations reported in this paper have to be considered as just an initial step in the quantification of turbulent effects on warm-rain processes in various cloud systems. First, assessment of the dynamical enhancement in the cloud field simulations requires more analysis, beyond what was presented here. We will continue to analyze the simulations and will present our results in the future. Second, as shown in Seifert et al. (2010), higher spatial resolution in LES simulations not only leads to a significantly different surface precipitation rate, but also to a different enhancement factor (see their Table II therein). This suggests that higher spatial resolution bin simulations of the type reported here should be considered. Moreover, work should continue to obtain and use improved formulations of the turbulent kernel and include effects of small-scale turbulence intermittency in LES simulations. Applying different formulations of the cloud microphysics (e.g., based on the Lagrangian approach, Andrejczuk et al., 2010) should also be considered to ensure that limitations of the bin microphysics approach play an insignificant role. One should also investigate the impacts in other ice-free cloud systems, such as subtropical stratocumulus. Precipitation processes in stratocumulus involve complicated and still poorly understood feedbacks (cf. Wood, 2012), and turbulence (although weaker than in shallow cumuli) may play a significant role. Finally, since simulated effects of cloud turbulence are dramatic, one should attempt to use remote sensing observations (either ground-based or from space) in an attempt to validate the impacts. All these aspects warrant additional investigations (some of them already in progress) and we plan to report them in forthcoming publications.

Acknowledgements. This work was supported by the NSF through grants OCI-0904534 and OCI-0904449. A. A. Wyszogrodzki was also supported by NSF grant OCI-0904599. WWG was partially supported by the NSF Science and Technology Center for Multiscale Modeling of Atmospheric Processes (CMMAP; managed by Colorado State University under cooperative agreement ATM-0425247) and by the DOE ASR grant DE-SC0008648. L.-P. Wang also acknowledges visitor support from the NCAR's Geophysical Turbulence Program (GTP). Computer time at NCAR was provided by NSF through MRI grants CNS-0421498, CNS-0420873, CNS-0420985, NSF sponsorship of the National Center for Atmospheric Research, the University of Colorado, and a grant from the IBM Shared University Research (SUR) program. National Center for Atmospheric Research is sponsored by the National Science Foundation.

Edited by: C. Hoose

\section{References}

Andrejczuk, M., Grabowski, W. W., Reisner, J., and Gadian, A.: Cloud-aerosol interactions for boundary-layer stratocumulus in the Lagrangian Cloud Model, J. Geophys. Res., 115, D22214, doi:10.1029/2010JD014248, 2010.

Ayala, O., Grabowski, W. W., and Wang, L.-P.: A hybrid approach for simulating turbulent collisions of hydrodynamicallyinteracting particles, J. Comput. Phys., 225, 51-73, 2007.

Ayala, O., Rosa, B., Wang, L.-P., and Grabowski, W. W.: Effects of turbulence on the geometric collision rate of sedimenting droplets: Part 1: Results from direct numerical simulation, New J. Phys., 10, 075015, doi:10.1088/1367-2630/10/7/075015, 2008a.

Ayala, O., Rosa, B., and Wang, L.-P.: Effects of turbulence on the geometric collision rate of sedimenting droplets: Part 2: Theory and parameterization, New J. Phys., 10, 075016, also Corrigendum, New J. Phys., 10, 099802, doi:10.1088/13672630/10/7/075016, 2008b.

Beard, K. V.: Terminal velocity and shape of cloud and precipitation drops aloft, J. Atmos. Sci., 33, 851-864, 1976.

Bott, A.: A positive definite advection scheme obtained by nonlinear renormalization of the advective fluxes, Mon. Weather Rev., 117, 1006-1015, 1998.

Chun, J., Koch, D. L., Rani, S. L., Ahluwalia, A., and Collins, L. R.: Clustering of aerosol particles in isotropic turbulence, J. Fluid Mech., 536, 219-251, 2005.

Cooper, W. A., Lasher-Trapp, S. G., and Blyth, A. M.: The influence of entrainment and mixing on the initial formation of rain in a warm cumulus cloud, J. Atmos. Sci., 70, 1727-1743, 2013.

Dávila, J. and Hunt, J. C. R.: Settling of small particles near vortices and in turbulence, J. Fluid Mech., 440, 117-145, 2001.

Franklin, C. N., Vaillancourt, P. A., Yau, M. K., and Bartello, P.: Collision rates of cloud droplets in turbulent flow, J. Atmos. Sci., 62, 2451-2466, 2005.

Grabowski, W. W.: Indirect impact of atmospheric aerosols in idealized simulations of convective-radiative quasi-equilibrium, J. Climate, 19, 4664-4682, 2006.

Grabowski, W. W.: Representation of turbulent mixing and buoyancy reversal in bulk cloud models, J. Atmos. Sci., 64, 36663690, 2007.

Grabowski, W. W. and Clark, T. L.: Cloud-environment interface instability: Rising thermal calculations in two spatial dimensions, J. Atmos. Sci., 48, 527-546, 1991.

Grabowski, W. W. and Clark, T. L.: Cloud-environment interface instability, part II: Extension to three spatial dimensions, J. Atmos. Sci., 50, 555-573, 1993.

Grabowski, W. W. and Wang, L.-P.: Diffusional and accretional growth of water drops in a rising adiabatic parcel: effects of the turbulent collision kernel, Atmos. Chem. Phys., 9, 2335-2353, doi:10.5194/acp-9-2335-2009, 2009.

Grabowski, W. W. and Wang, L.-P.: Growth of cloud droplets in a turbulent environment, Annu. Rev. Fluid Mech., 45, 293-324, 2013.

Grabowski, W. W., Thouron, O., Pinty, J.-P., and Brenguier, J.-L.: A hybrid bulk-bin approach to model warm-rain processes, J. Atmos. Sci., 67, 385-399, 2010. 
Grabowski, W. W., Andrejczuk, M., and Wang, L.-P.: Droplet growth in a bin warm-rain scheme with Twomey CCN activation, Atmos. Res., 99, 290-301, 2011.

Hall, W. D.: A detailed microphysical model within a twodimensional framework: model description and preliminary results, J. Atmos. Sci., 37, 2486-2507, 1980.

Holland, J. Z. and Rasmusson, E. M.: Measurements of the atmospheric mass, energy, and momentum budgets over a 500 kilometer square of tropical ocean, Mon. Weather Rev., 101, 44-55, 1973.

Jarecka, D., Grabowski, W. W., and Pawlowska, H.: Modeling of subgrid-scale mixing in large-eddy simulation of shallow convection, J. Atmos. Sci., 66, 2125-2133, 2009.

Jarecka, D., Grabowski, W. W., Pawlowska, H., and Wyszogrodzki, A. A.: Modeling of subgrid-scale cloud-clear air turbulent mixing in large eddy simulation of cloud fields, J. Phys. Conf. Ser., 318, 072010, doi:10.1088/1742-6596/318/7/072010, 2011.

Khain, A., Ovtchinnikov, M., Pinsky, M., Pokrovsky, A., and Krugliak, H.: Notes on the state-of-the-art numerical modeling of cloud microphysics, Atmos. Res., 55, 159-224, 2000.

Kolmogorov, A. N.: A refinement of previous hypotheses concerning the local structure of turbulence in a viscous incompressible fluid at high Reynolds number, J. Fluid Mech., 13, 82-85, 1962.

Margolin, L. G., Smolarkiewicz, P. K., and Sorbjan, Z.: Large-eddy simulations of convective boundary layers using nonoscillatory differencing, Physica D, 133, 390-397, 1999.

Pinsky, M. B. and Khain, A. P.: Collisions of small drops in a turbulent flow. Part II: Effects of flow accelerations, J. Atmos. Sci., 61, 1926-1939, 2004.

Pinsky, M. B., Khain, A. P., and Shapiro, M.: Collisions of small drops in a turbulent flow. Part I: Collision efficiency. Problem formulation and preliminary results, J. Atmos. Sci., 56, 25852600, 1999.

Pope, S. B.: Turbulent Flows, Cambridge University Press, Cambridge, UK, 771 pp., 2000.

Pruppacher, H. R. and Klett, J. D.: Microphysics of Clouds and Precipitation, Kluwer Academic, Kluwer Academic, Dordrecht, the Netherlands, 954 pp., 1997.

Prusa, J. M., Smolarkiewicz, P. K., and Wyszogrodzki, A. A.: EULAG, a computational model for multiscale flows, Comput. Fluids, 37, 1193-1207, 2008.

Rosa, B., Parishani, H., Ayala, O., Wang, L.-P., and Grabowski, W. W.: Kinematic and dynamic pair collision statistics of sedimenting inertial particles relevant to warm rain initiation, J. Phys. Conf. Ser., 318, 072016, doi:10.1088/17426596/318/7/072016, 2011.

Rosa, B., Parishani, H., Ayala, O., Wang, L.-P., and Grabowski, W. W.: Kinematic and dynamic collision statistics of cloud droplets from high-resolution simulations, New J. Phys., 15, 045032, doi:10.1088/1367-2630/15/4/045032, 2013.

Schumann, U.: Subgrid length-scales for large-eddy simulation of stratified turbulence, Theor. Comp. Fluid Dyn., 2, 279-290, 1991.

Seifert, A., Nuijens, L., and Stevens, B.: Turbulence effects on warm-rain autoconversion in precipitating shallow convections, Q. J. Roy. Meteor. Soc., 136, 1753-1762, doi:10.1002/qj.684, 2010.
Siebesma, A. P., Bretherton, C. S., Brown, A., Chlond, A., Cuxart, J., Duynkerke, P. G., Jiang, H., Khairoutdinov, M., Lewellen, D., Moeng, C.-H., Sanchez, E., Stevens, B., and Stevens, D. E.: A large eddy simulation intercomparison study of shallow cumulus convection, J. Atmos. Sci., 60, 1201-1219, 2003.

Slawinska, J., Grabowski, W. W., Pawlowska, H., and Morrison, H. Droplet activation and mixing in large-eddy simulation of a shallow cumulus field, J. Atmos. Sci., 69, 444-462, 2012.

Sreenivasan, K. R. and Antonia, R. A.: The Phenomenology of small-scale turbulence, Annu. Rev. Fluid Mech., 29, 435-472, 1997.

Sreenivasan, K. R. and Kailasnath, P.: An update on the intermittency exponent in turbulence, Phys. Fluids A-Fluid, 5, 512-514, 1993.

Stevens, B. and Seifert, A.: Understanding macrophysical outcomes of microphysical choices in simulations of shallow cumulus convection, J. Meteorol. Soc. Jpn., 86A, 143-162, 2008.

Van Zanten, M. C., Stevens, B. B., Nuijens, L., Siebesma, A. P., Ackerman, A., Burnet, F., Cheng, A., Couvreux, F., Jiang, H., Khairoutdinov, M., Kogan, Y., Lewellen, D. C., Mechem, D., Nakamura, K., Noda, A., Shipway, B. J., Slawinska, J., Wang, S., and Wyszogrodzki, A.: Controls on precipitation and cloudiness in simulations of trade-wind cumulus as observed during RICO, J. Adv. Model. Earth Syst., 3, M06001, doi:10.1029/2011MS000056, 2010.

Wang, L.-P. and Maxey, M. R.: Settling velocity and concentration distribution of heavy particles in homogeneous isotropic turbulence, J. Fluid Mech., 256, 27-68, 1993.

Wang, L.-P., Wexler, A. S., and Zhou, Y.: Statistical mechanical descriptions of turbulent coagulation of inertial particles, J. Fluid Mech., 415, 117-153, 2000.

Wang, L.-P., Ayala, O., Kasprzak, S. E., and Grabowski, W. W. Theoretical formulation of collision rate and collision efficiency of hydrodynamically-interacting cloud droplets in turbulent atmosphere, J. Atmos. Sci., 62, 2433-2450, 2005.

Wang, L.-P., Ayala, O., Xue, Y., and Grabowski, W. W.: Comments on "Droplets to drops by turbulent coagulation", J. Atmos. Sci., 63, 2397-2401, 2006a.

Wang, L.-P., Franklin, C. N., Ayala, O., and Grabowski, W. W. Probability distributions of angle of approach and relative velocity for colliding droplets in a turbulent flow, J. Atmos. Sci., 63 , 881-900, 2006b.

Wang, L.-P., Ayala, O., Rosa, B., and Grabowski, W. W.: Turbulent collision efficiency of heavy particles relevant to cloud droplets, New J. Phys., 10, 075013, doi:10.1088/1367-2630/10/7/075013, 2008.

Wood, R.: Stratocumulus clouds, Mon. Weather Rev., 140, 2373 2423, 2012.

Wyszogrodzki, A. A., Grabowski, W. W., and Wang, L.-P.: Activation of cloud droplets in bin-microphysics simulation of shallow convection, Acta Geophys., 59, 1168-1183, doi:10.2478/s11600-011-0052-y, 2011.

Xue, Y., Wang, L.-P., and Grabowski, W. W.: Growth of cloud droplets by turbulent collision-coalescence, J. Atmos. Sci., 65, 331-356, 2008.

Zaichik, L. I. and Alipchenkov, V. M.: Pair dispersion and preferential concentration of particles in isotropic turbulence, Phys. Fluids, 15, 1776-1787, 2003. 
Zaichik, L. I., Simonin, O., and Alipchenkov, V. M.: Two statistical models for predicting collision rates of inertial particles in homogeneous isotropic turbulence, Phys. Fluids, 15, 2995-3005, 2003.
Zhou, Y., Wexler, A. S., and Wang, L.-P.: Modeling turbulent collision of bidisperse inertial particles, J. Fluid Mech., 433, 77-104, 2001. 